\title{
Effects of Reynolds Number on Flow Mediated Interaction Between Two Cylinders
}

\author{
Zhonglu Lin ${ }^{1, *}$, Dongfang Liang ${ }^{1}$, and Ming Zhao ${ }^{2}$ \\ ${ }^{1}$ Engineering Department, Trumpington St, University of Cambridge, Cambridge, CB2 1PZ \\ ${ }^{2}$ School of Computing, Engineering and Mathematics, Western Sydney University, Penrith, \\ New South Wales, Australia \\ *Address all communications to this author at zl352@cam.ac.uk
}

\section{ABSTRACT}

This paper studies the interaction between two cylinders of an identical diameter immersed in quiescent fluid. The master cylinder carries out forced vibration, while the adjacent slave cylinder is elastically-mounted and has only one-degree-of-freedom along the centreline between the two cylinders. In this study, the geometry of the problem is fixed, with an initial gap ratio of the two cylinders of 0.9 and a non-dimensional vibration amplitude of the master cylinder of $0.47 \%$. In total, 7480 two-dimensional cases have been simulated to cover the parameter space of the problem, with the the Reynolds number ranging from 10 to 330, the structural damping factor of the slave cylinder ranging from 0 to 0.2, the mass ratio of the slave cylinder ranging from 1.5 to 2.5, and the master cylinder's oscillation frequency ratio ranging from 0.05 to 3.2. Both the resonance amplitude and resonance frequency are found to increase with the Reynolds number. A critical Reynolds number is discovered, beyond which the vibration centre of the slave cylinder drifts away from the master cylinder, but below which the vibration centre of the slave cylinder approaches the master cylinder. This effect 
is amplified when the master cylinder vibrates at a higher frequency ratio. Key words: flow around circular cylinders; flow induced vibration; resonance; computational fluid dynamics

\section{INTRODUCTION}

Flow mediated interaction is a phenomenon that objects moving in a fluid interact with the nearby objects via the perturbed fluid. The ensuing movement of the neighbouring objects is more complicated compared with the traditional scenarios with the objects under a steady or a periodic flow. The significance of studying flow mediated interaction relies on its wide occurrence in both natural [8-10, 12, 14, 23, 29] and artificial situations [1, 20, 28]. Extensive and elaborate studies have been carried out on this topic, for the purpose of comprehending the mechanism of the interactions. The revelations from these studies can thus be exploited in engineering applications $[2,27]$.

In this study, we focus on the flow mediated interaction between two cylinders immersed in a quiescent incompressible fluid. One cylinder, the master cylinder, carries out sinusoidal forced vibration to perturb the surrounding fluid, and the generated periodic flow thus interacts with the other cylinder, the slave cylinder, which is elastically-mounted with a damper. Despite the basic geometry, this problem involves a rich spectrum of physics and carries practical implications in many fields of research and engineering.

As the necessity to check the flow mediate interactions between multiple cylinders, various studies have already been conducted over the past decades to analyse the fluid-structure interactions regarding solely one periodically oscillating cylinder.

The scenario of a cylinder oscillating in a fluid is physically equivalent to a cylinder immersed in a oscillatory flow, which can be determined by two independent parameters, i.e. 
the Keulegan-Carpenter number and Reynolds number. The Keulegan-Carpenter number can be defined as $K C=U_{m} T / D$ [11], where $U_{m}$ is the amplitude of the oscillatory flow velocity, $T$ the period of oscillatory flow, and $D$ the diameter of the cylinder. Given sinusoidal flow, $K C=2 \pi A / D$. The Reynolds number is defined as $R e_{m}=U_{m} D / \nu$, where $\nu$ is the fluid kinematic viscosity.

In engineering application, the prediction of fluid-induced drag forces for a circular cylinder in oscillating or wave flow is predicted on the Morrison Equation [21], which represented the in-line force per unit length as

$$
F=\frac{1}{2} \rho D C_{D} U|U|+\frac{1}{4} \pi \rho D^{2} C_{m} \dot{U}
$$

where $\rho$ is the fluid density, $D$ is the body diameter and $U$ is the fluid velocity. $C_{m}=1+C_{a}$ is the inertia coefficient, and $C_{a}$ the added mass coefficient. $C_{D}$ is the drag coefficient. $C_{m}$ and $C_{D}$ are found to depend on both $K C$ [11] and $\beta=R e / K C$ [24]. Williamson [30] further studied the effects of the vortex motions on the forces upon a single cylinder in the range of $0<K C<35$ with $\beta$ fixed at 730 . He [30] reported reasonable symmetry at $K C<4$. Several regimes were classified according to the vortex patterns and $K C$. Specifically, at $0<K C<7$, a pair of small attached vortices are observed, which is similar to the flow pattern generated by the master cylinder in the present study. Based on this pattern, five flow regimes were defined according to $K C$. While Williamson [30] carried out experiments at a fixed $\beta$, Tatsuno and Bearman [25] further explored in the range of $K C<15$ and $\beta<160$. They classified eight flow regimes based on flow visualization, which were referred to as regimes $A^{*}, A, B, C, D, E, F, G$. The flows in regimes $A$ and $A^{*}$ are entirely two- 
dimensional (2D), whereas three-dimensional (3D) flow features are observed in regime $B$. Based on the useful flow regime map from Tatsuno and Bearman [25], Elston, Sheridan, and Blackburn $[5,6]$ further examined the symmetry breaking phenomenon, and identified the parameters related to the breakage of different types of symmetries for this case, including the onset of both 2D and 3D instability. It appears to be the first time that the flow fields of a single cylinder oscillating in quiescent fluid are studied in a quantified manner using direct numerical simulation and Floquet analysis, rather than rely purely on visual observation. In regime $A, A^{*}$, the flow fields are symmetrical regarding the in-line axis, whereas. The present study is within regimes $A, A^{*}$ and $B$, and for all the involved cases, the flow field is symmetric. Dutsch, Durst, Becker, and Lienhart [4] carried out laser Doppler anemometry measurements of a laminar flow generated by the harmonic oscillation of a circular cylinder in otherwise still water. By comparing with these experimental results, an in-house 2D Navier-Stokes model is validated by [16], which is the basic tool for the present study.

In contrast to the abundant research on the cylinders vibrating in a still fluid, the research of the flow mediated interaction between multiple immersed cylinders is relatively scarce. Lamb [13] studied the interaction between two spheres immersed in inviscid fluid. One sphere is forced to oscillate along the centre-line, whereas the other nearby sphere of neutral buoyancy responds freely to the disturbed fluid. By theoretical analysis, Lamb [13] stated that the free sphere is "on the whole" attracted towards the forcedly oscillating sphere due to the imbalanced pressure force. By both analytical and numerical methods, Nair and Kanso [22] studied an identical configuration in greater detail, but for the case of two circular cylinders rather than two spheres. One cylinder is started impulsively and is forced to oscillate along the centre-line between two cylinders, whereas the second responds freely. 
Nair and Kanso [22] discovered that the free cylinder can be either repelled away or attracted towards the forcedly oscillating cylinder, depending on the initial velocity direction of the oscillating cylinder. They further suggested that this should also be the case for the sphere scenario analysed by Lamb [13], who only captured the attraction.

The flow mediated interaction between two cylinders was further investigated by Gazzola, Mimeau, Tchieu, and Koumoutsakos [7], but the fluid was taken to be viscous rather than inviscid. They found a threshold Reynolds number, beyond which the slave or passive cylinder is repelled by the master or active cylinder, and under which it is attracted to the master cylinder. A secondary flow structure is discovered between the two cylinders. An increase in Reynolds number, i.e., a decrease in viscosity, slows down the dissipation of the secondary flow, which favours the repulsion of the slave cylinder by the master. They further discovered that the threshold Reynolds number is indifferent to the initial phase of the master, i.e., the direction of the initial velocity of the master cylinder. This conclusion is different from that drawn in Nair and Kanso [22] concerning the inviscid flow, where the initial phase of the movement governs the repulsion or attraction. With the increase in the initial gap, the threshold Reynolds number decreases exponentially, whereas it is less sensitive to the size difference between the two cylinders. Based on these observations, Gazzola, Mimeau, Tchieu, and Koumoutsakos [7] concluded that the flow features have a greater influence than the inertia of the slave cylinder. As a result, in the present study, the diameters of the slave and the master cylinders are identical. They also found that, given a very small vibration amplitude of the master cylinder, the level of repulsion or attraction is significantly reduced. Therefore, in the present study, the master cylinder oscillates at an amplitude of $0.477 \mathrm{D}$, i.e. $K C=3$, allowing convenient observation and study of the 
repulsion and attraction.

Lin, Liang and Zhao $[17,18]$ investigated a similar case. One cylinder is forced to oscillate along the centre-line between two cylinders, whereas the second one is elastically-mounted. They found that, for sufficiently small amplitude of the master cylinder, the response of the slave cylinder is essentially sinusoidal and it is sensitive to the master cylinder's vibration frequency, while the master cylinder's amplitude does not play an important role.

In summary, a large amount of research has been carried out on the flow past a single or multiple solid objects, but there is only limited research on the flow mediated interaction between immersed objects. Furthermore, the previous research [15-18] considers the case where the Reynolds number keeps a constant. This research aims to investigate the problem in a large parameter space, with a particular focus on the Reynolds number influence.

\section{PROBLEM SETUP AND NUMERICAL METHOD}

In this study, two identical rigid cylinders are immersed in otherwise still fluid, as seen in Fig. 2. At time zero, the master cylinder starts to vibrate harmonically to disturb the fluid, whereas the slave cylinder, which has 1 degree of freedom (1DOF) along the y axis, vibrates correspondingly under the action of the imbalanced hydrodynamic force. The nondimensional analysis shows that it requires six non-dimensional parameters to define the problem, i.e. gap ratio $G / D$, frequency of the master cylinder $f_{1} / f_{n}$, amplitude of the master cylinder $A_{1} / D$, the mass ratio of the slave cylinder $m^{*}=m_{c} / m_{d i s}$, damping factor of the slave cylinder $\zeta$, and Reynolds number based on the maximum velocity of the master cylinder $R e_{m}=U_{m} D / \nu=2 \pi A_{1} f_{1} D / \nu$. Here, $G$ is the initial gap distance between the two cylinders, $f_{1}$ is the vibration frequency of the master cylinder, $f_{n}=(1 / 2 \pi) * \sqrt{k / m_{c}}$ is the structural 
natural frequency of the slave cylinder in vacuum, $k$ is the stiffness of the spring, $m_{c}$ the mass of the slave cylinder, $m_{\text {dis }}$ the mass of the fluid displaced by the slave cylinder, and $\nu$ the kinematic viscosity coefficient of the fluid.

Simulations are conducted for a range of combinations of parameters. In summary, the Reynolds number $R e_{m}$ varies from 10 to 150; The master cylinder's frequency $f_{1} / f_{n}$ ranges from 0.05 to 3.2 ; the mass ratio $m^{*}$ takes the value of $1.5,1.7,2.0,2.2$ or 2.5 ; the amplitude of the master cylinder $A_{1} / D$ is fixed at 0.477 ; the gap ratio $G / D$ is a constant value of 0.9 . The Keulegan-Carpenter number and the Stokes number of the master cylinder can then be calculated to be $K C=2 \pi A_{1} / D=3$ and $\beta=R e_{m} / K C=3.3 \sim 110$, respectively. In total, 7480 combinations of parameters are examined. The tested frequency of the master cylinder's vibration $f_{1} / f_{n}$ covers all the resonating frequencies of the slave cylinder's response. The range of Reynolds number $R e_{m}$ covers both the repelling and attraction regimes for the slave cylinder. The initial gap ratio and the amplitude of the master cylinder are fixed to limit the complexity of the studied problem. Hence, the flow considered in this study is within regime $A^{*}$ and $A$. These selections of parameters ensure the validity of the 2D Navier-Stokes simulations. If the master cylinder's Keulegan-Carpenter number $K C$ and Stokes number $\beta$ are too large, the flow may become irregular and the slave cylinder's response may undergo bifurcations to become unstable, which is beyond the scope of the present analyses. These low mass ratios are typical for engineering structures immersed in water, e.g. the mass ratio of concrete in water is about 2.5 .

The two-dimensional Navier-Stokes equations are solved by the Petrov-Galerkin finiteelement method (PG-FEM). The moving cylinder boundaries are handled through the use of an arbitrary Lagrangian-Eulerian (ALE) scheme. In the governing equations, the length, 
time, velocity and pressure are non-dimensional quantities according to:

$$
x_{i}^{*}=\frac{x_{i}}{D}, t^{*}=f_{n} t, u_{i}^{*}=\frac{u_{i}}{f_{n} D}, p^{*}=\frac{p}{\rho f_{n}^{2} D^{2}}
$$

where $x_{1}^{*}=x / D$ and $x_{2}^{*}=y / D$ are the Cartesian coordinates as shown in figure 2. $t$ is time, $f_{n}$ is the structural natural frequency in vacuum determined by the slave cylinder's mass and spring stiffness as explained before, $u_{i}$ is the fluid velocity component in the $x_{i}$ direction, and $p$ is the pressure. The variables with stars represent the non-dimensional quantities. The non-dimensional incompressible two-dimensional Navier-Stokes equations in the ALE framework are

$$
\begin{gathered}
\frac{\partial u_{i}^{*}}{\partial x_{i}^{*}}=0, \\
\frac{\partial u_{i}^{*}}{\partial t^{*}}+\left(u_{j}^{*}-u_{j, \text { mesh }}^{*}\right) \frac{\partial u_{j}^{*}}{\partial x_{j}^{*}}+\frac{\partial p^{*}}{\partial x_{i}^{*}}=\frac{U_{m}}{R e_{m} f_{n} D} \frac{\partial^{2} u_{i}^{*}}{\partial x_{j}^{*} \partial x_{j}^{*}}
\end{gathered}
$$

where $u_{j, m e s h}^{*}$ is the velocity of the mesh movement.

The motion equations for the master and the slave cylinders are

$$
\begin{gathered}
Y_{1}=A_{1} \sin \left(2 \pi f_{1} t\right) \\
\frac{\partial^{2} Y_{2}}{\partial t^{2}}+4 \pi f_{n} \zeta \frac{\partial Y_{2}}{\partial t}+4 \pi^{2} f_{n}^{2} Y_{2}=\frac{2}{\pi} \frac{U_{m}^{2}}{m^{*} D} C_{Y 2}
\end{gathered}
$$

where $Y_{1}$ and $Y_{2}$ are the displacements of the master and slave cylinders, respectively, on 
the $y$ direction, $\zeta$ is the damping factor, $C_{Y 2}=F_{Y 2} /\left(0.5 \rho D U_{m}^{2}\right)$ is the force coefficient for the slave cylinder and $F_{Y 2}$ is the force acting on the slave cylinder in the y direction.

Mesh independence study has been conducted with a variety of mesh density as listed in Table 1. Based on these meshes, typical cases were tested as seen in Fig. 4. It can be shown that the mesh with normal density can already provide a decent accuracy. In order to be conservative, we chose the dense mesh to run all the simulations in this paper.

The numerical model presented above has been extensively validated in many previous studies regarding the flow around circular cylinders $[3,15,16,19,26,31]$.

\section{ANALYSIS OF SLAVE CYLINDER'S MOVEMENT}

In the present study, we focus on the effects of Reynolds number $R e_{m}$ on the interactions between the two cylinders. For the cases discussed in this section, turbulence does not occur, while the flows are laminar and symmetric, corresponding to flow regimes $A$ and $A^{*}[25]$. After a few initial periods of oscillation, the vibration of the slave cylinder actuated by the flows is steady, regular and repetitive.

Reynolds number significantly affects the interactions between the two cylinders, and thus the displacement of the slave cylinder. In general, with the increase of the Reynolds number, both the resonance vibration amplitude and the resonance frequency is increased, while the phase difference between the master and the slave cylinder is reduced. A threshold Reynolds number is discovered, beyond which the slave cylinder vibrates steadily about a position that is closer to the master cylinder than the initial stationary position, and below which the slave cylinder is repelled away from the master cylinder. This is similar to the critical Reynolds number discussed in Case B in [7]. The difference is that in the current 
312

scenario, the slave cylinder is constrained by a spring rather than being free, so it is not carried away by the flowing fluid but vibrates about a constant position away from the initial position. The influence of the damping factor and the mass ratio on the relationship between the Reynolds number and the slave cylinder's displacement is also examined. We then further discuss the influence of the Reynolds number on the flow field adjacent to the two cylinders.

\section{Effects of Reynolds Number on Slave Cylinder's Vibration Amplitude}

The amplitude spectra of slave cylinder's displacement offer an comprehensive overview to the dynamic characteristics of the slave cylinder, as depicted in Fig. 5. It is seen that the amplitude of the dominant frequency components at resonance increases exponentially with $R e_{m}$. As seen in Fig. 5a, a series of frequency components are observed with almost zero component frequencies $f / f_{n} \approx 0$. This indicates that the vibration centre of the slave cylinder at the periodic state shifts from its initial position, giving rise to a component with an extremely low $f / f_{n}$. It is also seen that the component amplitude at zero frequency increases with master cylinder's oscillation frequency $f_{1} / f_{n}$, which means when the master cylinder vibrates at a higher frequency, the slave cylinder drifts further away from the initial position. The influence of the Reynolds number on the component amplitude at zero frequency is more complicated. It initially decreases with $R e_{m}$ at low Reynolds numbers and, when $R e_{m}$ goes beyond a critical value, e.g. $R e_{m}=50$ in Fig. 5 c, the amplitude increases with $R e_{m}$ again. The relationship between the Reynolds number and the vibration centre drift of the slave cylinder will be further discussed in Section 3.

The slave cylinder's resonance amplitude and the resonance frequency both increase with 
the Reynolds number, as seen in Fig. 6, which matches the pattern shown in Fig. 5. At $f_{1} / f_{n}<1$, the slave cylinder's vibration amplitude generally increases with Reynolds number, whereas at $f_{1} / f_{n}>1$ the amplitude decreases with Reynolds number, as demonstrated in Fig. 6a. As seen in Fig. 6b, when $R e_{m}$ increases from 10 to 150, the resonance amplitude doubles from 0.3 to 0.6 . Meanwhile, the resonance frequency increases from 0.625 to 0.74 . In terms of secondary resonance at $0.34<f_{1} / f_{n}<0.4$, as $R e_{m}$ rises from 30 to 150 , the amplitude of the secondary resonance doubles from 0.075 to 0.14 and the secondary resonance frequency rises from 0.36 to 0.375 . The situation at $R e_{m}=10$ is special that the secondary resonance is not observed, and, here, the amplitude at $R e_{m}=10$ is higher than those at $R e_{m}=30-50$. The vibration phase difference between the two cylinders is demonstrated in Fig. 6c. Overall, the increase of $R e_{m}$ causes the phase difference curves to shift upwards. The steepness of the phase change at resonance slightly increases with $R e_{m}$ as well.

A critical damping factor is discovered at $\zeta=0.1$, below which the resonance amplification factor increases with the Reynolds number, and beyond which the factor decreases with the Reynolds number, as seen in Fig. 7a. It is also seen that the increase of the Reynolds number amplifies the effect of the damping factor. In other words, the peak amplification factor becomes more sensitive to the damping factor at a high Reynolds number. The same pattern applies to the secondary resonance regime as shown in the inset in Fig. 7b, although the secondary peak amplification factor has a critical damping factor of $\zeta=0.025$, rather than $\zeta=0.1$ for the primary resonance peak in this case. The effects of the damping factor are weakened at very low or very high frequencies, which is consistent with the conclusion made in [17]. The mechanism of the critical damping factor will be discussed in detail later. Reynolds number does not qualitatively affect the relationship between the mass ratio 
and the peak amplification factor, as seen in Fig. 8. The primary and secondary resonance frequencies increase with the mass ratio, regardless of the Reynolds number.

\section{Effects of Reynolds Number on Slave Cylinder's Vibration Centre Drift}

Similar to Case B in [7], the slave cylinder in the presented cases is also observed to be repelled away or attracted towards the master cylinder, depending on the value of $R e_{m}$. Nevertheless, it should be noted that the repelling and attraction discussed is referred to the centre of the slave cylinder's vibration relative to its stationary position at time zero - either closer or further away from the master cylinder. For this reason, in the following discussion, we address this phenomenon as "vibration centre drift".

At a very low Keulegan-Carpenter number of the master cylinder $K C=0.157 \sim 0.628$ and a fixed Reynolds number of $R e_{m}=100$, the phenomenon of vibration centre drift was hardly observed in the previous studies $[17,18]$. However, when the $K C$ is increased to 3 and a variety of $R e_{m}$ from 10 to 150 is tested, the vibration centre drift of the slave cylinder is clearly observed. Here, based on the assumption that the flow mediated interactions is periodical, the amplitude of the slave cylinder's vibration is calculated as $A_{2}=\left(Y_{\max }-\right.$ $\left.Y_{\min }\right) / 2$, where $Y_{\max }$ and $Y_{\min }$ are the maximum and the minimum, respectively, of the slave cylinder displacement $Y_{2}$ in the last 50 periods of steady oscillations. The simulations were run for at least 60 periods. The vibration centre drift of the slave cylinder is calculated as $\Delta \bar{Y}_{2}=\left(Y_{\max }+Y_{\min }\right) / 2$

A critical Reynolds number is discovered that, beyond which the slave cylinder is repelled away from the master cylinder, and below which the the slave cylinder is attracted towards the master cylinder. For example, as seen in Fig. 9a, the critical Reynolds number is at 
about $R e_{m}=40$, when the frequencies are relatively high at $f_{1} / f_{n}>1$. The variation of the vibration centre drift demonstrates a unique pattern at both the secondary and the primary resonance regimes at $0.25<f_{1} / f_{n}<0.85$, as seen in Fig. 9b. The two drops in the curves at $f_{1} / f_{n}=0.35$ and at $f_{1} / f_{n}=0.7$ correspond to the secondary and primary resonance regimes, respectively. This indicates that, when resonance occurs, the slave tends to be attracted towards the master cylinder. On the contrary, when the master cylinder oscillates at a relatively high frequency at $f_{1} / f_{n}>1$, the resonance-induced drop in the curve is no longer observed. An increased frequency is found to amplify the effects of $R e_{m}$ on the repelling or the attraction of the slave cylinder. Figure $9 \mathrm{c}$ is a close-up at the primary resonance at $f_{1} / f_{n}=0.64-0.8$, where the attraction effect due to the resonance is clearly identified. In addition, the curves for $R e_{m} \geq 50$ converge at the point of $f_{1} / f_{n}=0.78, A_{2} / A_{1}=0.01$. This indicates that, at $R e_{m} \geq 50$, when the master cylinder vibrates at the frequency of $f_{1} / f_{n}=0.78$, the distance of the vibration centre drift is constantly $\Delta \bar{Y}_{2}=0.01 A_{1}$, being independent of the Reynolds number. Nevertheless, at $R e_{m}=10$, this pattern of convergence is not observed. Moreover, the information in Fig. 9c can be presented in another form. $\Delta \bar{Y}_{2} / A_{1}$ can be plotted against $R e_{m}$ rather than $f_{1} / f_{n}$, as shown in Fig. 9. At a high frequency $f_{1} / f_{n} \geq 1$, the slave cylinder is increasingly repelled from the master cylinder with the rise of the Reynolds number, especially at $10<R e_{m}<100$. At $R e_{m}>100$, the vibration centre drift $\Delta \bar{Y}_{2}$ becomes insensitive to the Reynolds number. A convergence point is observed at $R e_{m}=50$ and $\Delta \bar{Y}_{2} / A_{1}=0.01$, which means that the vibration centre drift of the slave cylinder is independent of the master cylinder's vibration frequency at $R e_{m}=50$ and $f_{1} / f_{n}>1$. Also, it is notable that the critical Reynolds number, i.e. the $R e_{m}$ corresponding to zero vibration centre drift, slightly increases with the master cylinder's 
vibration frequency.

The influence of the damping factor upon the slave cylinder's vibration centre drift is most significant at the resonance regime at $f_{1} / f_{n}=0.3-0.8$, but is much less influential at low and high frequencies at $f_{1} / f_{n}<0.25$ and $f_{1} / f_{n}>1$, as seen in Fig. 10a. In other words, at resonance, the slave cylinder tend to be attracted towards the master cylinder with a high damping factor. This pattern corresponds well to the damping factor's effect on the slave cylinder's responding amplitude, where the damping effect also becomes most significant at the resonance regimes and diminishes at both very high and very low frequency regimes, as demonstrated in [17]. As seen in Fig. 10b, it is interesting that a higher damping factor can actually push the slave cylinder further away from the initial vibration centre, which is counter-intuitive.

The Reynolds number affects the damping factor's influence on the frequency-amplitude relationship, as seen in Fig. 10a. In general, at the resonance regime, the vibration centre drift is more sensitive to damping factor with a higher Reynolds number. For example, as seen in Fig. 10b, with the damping factor $\zeta$ rising from 0 to 0.2 , the vibration centre drift $\Delta \bar{Y}_{2}$ increased by 0.003 from -0.023 to -0.020 at $R e_{m}=10$, whereas it increases by 0.012 from -0.008 to 0.004 at $R e_{m}=70$. The increment at $R e_{m}=10$ is only $25 \%$ of that at $R e_{m}=70$

Mass ratio $m^{*}$ also plays an important role on the vibration centre drift of the slave cylinder. In contrast to the damping factor, the mass ratio becomes more influential over the vibration centre drift when the frequency is beyond the resonance regime, as seen in Fig. 11a. With a further examination, we found that the correlation between the mass ratio and the vibration centre drift reverses twice at $R e_{m}=10$, but it reverses for only once 
at $R e_{m}=70$, as seen in Fig. 11b. For cases with low $R e_{m}=10$, vibration centre drift is positively correlated with $m^{*}$ at low frequencies. As $f_{1} / f_{n}$ goes beyond 0.5 , the correlation is reversed that the vibration centre drift decreases with $m^{*}$. When $f_{1} / f_{n}$ is further increased beyond 0.8 , the correlation is reversed back to be positive again, and remains positive for larger frequencies. Here, for $R e_{m}=10$, the correlation is reversed twice at $f_{1} / f_{n}=0.5$ and $f_{1} / f_{n}=0.8$, and it ends up in positive correlation. For cases with high $R e_{m}=70$, vibration centre drift is positively correlated with $m^{*}$ at low frequencies, similar to low $R e_{m}$ cases. When $f_{1} / f_{n}$ goes above 0.7 , the correlation becomes negative, and it remains negative for all frequencies at $f_{1} / f_{n}>0.7$. Here, the correlation is reversed once, and it ends up in negative correlation.

In summary, at a low frequency $f_{1} / f_{n}<0.5$ or a high frequency $f_{1} / f_{n}>0.8$, with the increase of the mass ratio, the slave cylinder is dragged towards its initial position, where $\Delta \bar{Y}_{2} / A_{1}=0$. However, at the primary resonance regime $0.5<f_{1} / f_{n}<0.8$, the increase of mass ratio may cause the slave cylinder to be attracted towards the master cylinder, which is counter-intuitive.

\section{FLOW FIELDS AROUND THE TWO CYLINDERS}

In this subsection, we examine the effect of the Reynolds number upon the flow fields surrounding the two cylinders. The typical cases with parameter combinations of $G / D=$ $0.9, A_{1} / D=0.477, m^{*}=1.5, f_{1} / f_{n}=2.8$ and various $R e_{m}$ are examined in detail. The major flow feature presented below is representative to other cases examined in this study. The flow resulting from the flow mediated interaction between the two cylinders moving 
along the vertical $(y)$ axis has the following symmetry properties:

$$
\begin{gathered}
u_{1}(x, y, t)=-u_{1}(-x, y, t) \\
\left(u_{1}, u_{2}\right)(x, y, t)=\left(u_{1}, u_{2}\right)(x, y, t+T)
\end{gathered}
$$

where Eq. 7 represents reflection symmetry about y-axis, while Eq. 8 stands for the periodic nature of the flow mediated interaction in the current range of parametric space. Since the scenario is symmetric regarding y-axis, Figs. 12 and 13 present only the left half of the region around the two cylinders.

With the increase of the Reynolds number, more vortices are generated. For example, pressure coefficient and velocity vectors in the gap at a high frequency $f_{1} / f_{n}=2.8$ with a constant phase of $\phi_{1}$ are plotted in Fig. 12, where the pressure coefficient is calculated as $C_{p}=p^{*} /\left[2 \pi\left(A_{1} / D\right)\left(f_{1} / f_{n}\right)\right]^{2}=p /\left(\rho U_{m}^{2}\right)$. At $R e_{m}=10$, no vortex is generated, as seen in Fig. $12 \mathrm{a}$, whereas at $R e_{m}=50$, a pair of vortices are generated in the gap, as shown in Fig. 12b. At a relatively high Reynolds number $R e_{m}=150,2$ pairs of vortices are generated in the gap, and another pair of vortices is observed at the far side of the master cylinder, as demonstrated in Fig. 12c. Although vortices are observed, vortex shedding does not occur at $R e_{m}=10-150$, and the vortices are dissipated before they can be shed away from the two cylinders.

The pressure decreases at the near side of the master cylinder with the increase in the Reynolds number, because the generated vortices help reduce pressure. The increase of Reynolds number can also be interpreted as the relative reduction of viscosity, causing the 
fluid to flow out of the gap more easily and thus more rapidly reducing the pressure accumulated in the gap. Also, the vibration amplitude of the slave cylinder decreases with Reynolds number, corresponding to the reduced pressure fluctuation surrounding the slave cylinder.

The fluid around the slave cylinder tends to flow upwards with the progression of Reynolds number at $\phi_{1}=180^{\circ}$, as seen in Fig. 12a1, $12 \mathrm{~b} 1$ and $12 \mathrm{c} 1$. The increase of the Reynolds number, i.e. the decrease of viscous influence, contributes to the slow dissipation of the secondary vortices at the near side of the slave cylinder in Fig. 12c1, which causes the fluid to flow upwards, favouring the repelling of the slave cylinder. This explains the increase of the vibration centre drift with the Reynolds number as seen in Fig. 9d. This mechanism is very similar to the cases where the slave cylinder is not constrained by a spring [7, p. 14].

At the resonance regime, as seen in Fig. 13, less vortices are generated in the gap between the two cylinders, compared with the high frequency situation demonstrated in Fig. 12. At $R e_{m}=150$, only one pair of vortices are generated in the gap at the resonance frequency, as shown in Fig. 12c1, while two pairs are observed at the high frequency as demonstrated in Fig. 13c. By comparing the velocity fields in Figs. 12 and 13, it is seen that the fluid flows more violently at a higher frequency, because the maximum velocity of the vibrating master cylinder $U_{m}$ is increased, contributing more dynamic energy imparted to the surrounding fluid and thus amplifying attraction and repelling of the slave cylinder.

The secondary vortices at the near side of the slave cylinder is observed at the high frequency as seen in Fig. 12c1 but it is not seen at the resonance frequency Fig. 13c. The reduction on the master cylinder's oscillation frequency weakens the secondary vortices in the gap, and thus reducing the repelling effect on the slave cylinder. This is consistent with the pattern shown in Fig. 9a. 
Pressure coefficient and streamlines at a high frequency $f_{1} / f_{n}=2.8$ are demonstrated in Fig. 14. With the increase of Reynolds number, the intensity of pressure fluctuation in the gap and at the far side of the slave cylinder both decreases, as seen in Fig. 14a1, 14a2 and 14a3. Since the pressure difference on the circumference of the slave cylinder is the main driver of its motion [17], the vibration amplitude of the slave cylinder is reduced due to the increase in the Reynolds number. This confirms the results shown in Fig. 6, where the amplification factor decreases with the Reynolds number. Also, with the increase of $R e_{m}$, the influence of vortices on the pressure is strengthened. This effect is indicated in Fig. 14a3 as the two small local low pressure spots located symmetrical beneath the master cylinder and in Fig. 14c3 as the two small local low pressure spots located symmetrical above the master cylinder.

The vorticity drawings, Figs. 15 and 16, demonstrate the flow pattern difference between the case with $R e_{m}=10$ lower than the critical Reynolds number and the case with $R e_{m}=150$ higher than the critical Reynolds number. In Fig. 15, we can see the difference when $R e_{m}$ is increased from 10 to 150 , which means the viscosity of the fluid is reduced.

At $R e_{m}=10$, the vortices are in general much larger than those at $R e_{m}=150$ due to high viscosity. As demonstrated in Fig. 16a, at $\phi=180^{\circ}$, the large vortices drive the surrounding fluid to flow downwards. The slave cylinder is immersed in the downward flow, and it is driven towards the master cylinder. The high viscosity also leads to quicker dissipation of the vortices. At $R e_{m}=10$ extra pair of vortices is never observed in the periodical vibration of the slave cylinder.

At $R e_{m}=150$, low viscosity causes the vortices in the gap harder to dissipated, and the size of vortex also becomes smaller. See Fig. $15 \mathrm{c} 2$ for example, the pair of vortices in the gap 
can be seen throughout the whole cycle, see Fig. 15a2-15d2, of the flow mediated interaction. This pair of vortices has opposite signs. The positive vortex is always on the left, whereas the negative vortex is always on the right. Together they propel a fluid flow to push away the slave cylinder. The flow structure are demonstrated by the streamlines in Fig. 16b. The existence of these vortices throughout the periodical vibration of the salve cylinder causes its vibration centre to drift towards the far side.

At $R e_{m}=10$, the vortices are in general much larger than those at $R e_{m}=150$ due to high viscosity. As demonstrated in Fig. 16a, at $\phi=180^{\circ}$, the large vortices drive the surrounding fluid to flow downwards, producing a strong attraction towards the master cylinder. The slave cylinder is immersed in the downward flow, and it is dragged towards the master cylinder. The high viscosity also leads to quicker dissipation of the vortices. At $R e_{m}=10$ extra pair of vortices is never observed in the periodical vibration of the slave cylinder, favouring the attraction of the slave cylinder.

In summary, at $R e_{m}=10$, the elastically-mounted slave cylinder's vibration centre drifts towards the master cylinder, whereas at $R e_{m}=150$, the flow pattern favours the repelling of the slave cylinder. Also, at around $R e_{m} \approx 60$, where the effects contributing to repulsion and attraction are almost equal and the vibration centre of the slave cylinder stays at the initial position.

As previously discussed, we define the critical damping factor as the damping factor below which the the slave cylinder's resonance amplitude increases with $R e_{m}$ and beyond which the amplitude decreases with $R e_{m}$.

The time histories of the hydraulic force coefficient show that the amplitude of the shear force decreases with $R e_{m}$ due to the decreasing viscosity. The pressure force amplitude rises 
with the $R e_{m}$ at $\zeta=0$. The increase in pressure force overweights the decrease in the shear force, so the amplitude of the force upon the cylinder increases as well. Therefore, vibration amplitude increases with $R e_{m}$ at $\zeta=0$. At $\zeta=0.2$, the shear force drops with $R e_{m}$, whereas the pressure force remains almost constant with the increase of $R e_{m}$. Consequently, the amplitude of the force upon the cylinder reduces. As a result, the vibration amplitude decreases with $R e_{m}$ at $\zeta=0$. This applies for both the primary resonance, Fig. 17a-17f, and secondary resonance, Fig. 17g-171.

The primary resonance, the pressure coefficient and velocity vector plots, Figs. 18 to 21, demonstrate that, at $\zeta=0$, the intensity of pressure fluctuation around the slave cylinder increases with $R e_{m}$, whereas, at $\zeta=0.2$, the intensity decreases with $R e_{m}$.

For example, as seen in Fig. 18b1-18b3, at $\zeta=0$, the positive pressure above the slave cylinder increases as $R e_{m}$ goes up from $R e_{m}=10$ to $R e_{m}=110$, whereas the negative pressure below the slave decreases only slightly. Similar patterns can be observed in other sub-figures in Fig. 18. So, at $\zeta=0$, the pressure fluctuation, in general, is strengthened. This is coherent to the time histories of the pressure coefficient shown in Fig. 17a-17c, where the pressure force upon the slave fluctuates with less amplitude while $R e_{m}$ goes up.

At $\zeta=0.2$, as shown in Fig. 19b1-19b3, the positive pressure above the slave cylinder remains almost constant with the variation of $R e_{m}$, while the negative pressure below the slave cylinder is weakened with the increase of $R e_{m}$. Together with other sub-figures in Fig. 19, it is clear that the pressure fluctuation is weakened due to the increase of $R e_{m}$. This corresponds to the decrease in the amplitude of the pressure force with $R e_{m}$ as seen in Fig. 17d-17f. Similar pattern can be found for the primary resonance pressure coefficient contours and velocity vectors shown in Figs. 20 and 21. 


\section{CONCLUSIONS}

We conducted a large number of numerical simulations to study the flow mediated interactions between two cylinders immersed in an otherwise still fluid. The master cylinder undergoes forced vibration with prescribed amplitude and frequency, whereas the slave cylinder is elastically-mounted with a damper. This paper focused on the effect of the Reynolds number on the responding slave cylinder.

Both the slave cylinder's resonance amplitude and resonance frequency are found to increase with the Reynolds number. The phase difference between the slave and the master decreases with Reynolds number. A critical damping factor is discovered, below which the resonance amplitude increases with the Reynolds number and beyond which the amplitude decreases with the Reynolds number. The increase of the mass ratio shifts the vibration amplitude curves to high-frequency region, but the mass ratio does not affect the overall trend of the relationship between the Reynolds number and the slave cylinder's vibration.

In terms of the vibration centre drift, a critical Reynolds number is discovered, beyond which the slave cylinder is repelled away from the master cylinder and below which the the slave cylinder is attracted towards the master cylinder. The existence of the critical Reynolds number is particularly obvious at high frequencies $f_{1} / f_{n}>1$, while at the resonance regime this pattern is not significant. At the secondary and the primary resonance regimes, the slave cylinder tends to be attracted towards the master cylinder. When the Reynolds number is greater than $R e_{m}=100$, the change of $R e_{m}$ can hardly cause the vibration centre drift of the slave cylinder. At the resonance regime, the slave cylinder is increasingly attracted towards the master cylinder with the increase of the damping factor. This effect can be amplified at small Reynolds numbers. The damping factor does not significantly influence the vibration 
centre drift at high frequencies $f_{1} / f_{n}>1$. With the increase in the mass ratio, the slave cylinder keeps approaching to its initial position. Contrary to the influence of the damping factor, the effect of the mass ratio grows with the increase of $f_{1} / f_{n}$.

The increase of the Reynolds number can result in more and stronger vortices to be generated in the gap. At high Reynolds numbers, the secondary vortices in the gap are generated, causing the fluid to flow towards the slave cylinder, contributing to the repelling force on the slave cylinder. The increase of the frequency causes the master cylinder to vibrate more violently, and thus amplifying both the repelling and the attracting action on the slave cylinder.

At resonance, the increase of Reynolds number leads to a lowered amplitude for shear force and a larger amplitude for pressure force. At $\zeta=0$, the increase in pressure force amplitude can overweight the drop in shear force amplitude, thus causing a resonance amplitude positively correlated to $R e_{m}$. Whereas at $\zeta=0.2$, this is not the case, resulting in a negative correlation between resonance amplitude and $R e_{m}$. This explains why the critical damping factor exists.

\section{ACKNOWLEDGEMENTS}

The study was primarily funded by Fuzhou Nuocheng Construction Project Management Ltd and the National Natural Science Foundation of China (Grant Nos. 51479111 and $51628901)$.

This work used the Cirrus UK National Tier-2 HPC Service at EPCC (http://www.cirrus.ac.uk) funded by the University of Edinburgh and EPSRC (EP/P020267/1). The computing at Cirrus is funded by EPSRC Tier-2 Open Access Call. 
This work used the ARCHER UK National Supercomputing Service (http://www.archer.ac.uk).

This work used the Cambridge Service for Data Driven Discovery (https://www.hpc.cam.ac.uk) hosted by the Research Computing Services at the University of Cambridge.

We are very grateful for the excellent technical support from the team at the University of Cambridge's Research Computing Services.

\section{REFERENCES}

Bampalas, N. and Graham, J. M. R. (2008). "Flow-Induced Forces Arising during the Impact of Two Circular Cylinders." Journal of Fluid Mechanics, 616, 205.

Bernitsas, M. M., Raghavan, K., Ben-Simon, Y., and Garcia, E. M. H. (2008). "Vivace (Vortex Induced Vibration Aquatic Clean Energy): a New Concept in Generation of Clean and Renewable Energy from Fluid Flow." Journal of Offshore Mechanics and Arctic Engineering, 130(4), 041101.

Cui, Z., Zhao, M., and Teng, B. (2014). "Vortex-Induced Vibration of Two Elastically Coupled Cylinders in Side-By-Side Arrangement." Journal of Fluids and Structures, 44, $270-291$.

Dütsch, H., Durst, F., Becker, S., and Lienhart, H. (1998). "Low-Reynolds-Number Flow around an Oscillating Circular Cylinder at Low Keulegan-Carpenter Numbers." Journal of Fluid Mechanics, 360, 249-271.

Elston, J. R., Blackburn, H. M., and Sheridan, J. (2006). "The Primary and Secondary Instabilities of Flow Generated by an Oscillating Circular Cylinder." Journal of Fluid Mechanics, 550, 359 . 
Elston, J. R., Sheridan, J., and Blackburn, H. M. (2004). "Two-dimensional floquet stability analysis of the flow produced by an oscillating circular cylinder in quiescent fluid." European Journal of Mechanics, B/Fluids, Vol. 23, 99-106.

Gazzola, M., Mimeau, C., Tchieu, A. A., and Koumoutsakos, P. (2012). "Flow Mediated Interactions between Two Cylinders at Finite Re Numbers." Physics of Fluids, 24(4), 043103.

Gazzola, M., Tchieu, A. A., Alexeev, D., de Brauer, A., and Koumoutsakos, P. (2016). "Learning to School in the Presence of Hydrodynamic Interactions." Journal of Fluid Mechanics, 789, 726-749.

Gyrya, V., Aranson, I. S., Berlyand, L. V., and Karpeev, D. (2010). "A Model of Hydrodynamic Interaction between Swimming Bacteria." Bulletin of Mathematical Biology, 72(1), $148-183$.

Ishikawa, T., Simmonds, M. P., and Pedley, T. J. (2006). "Hydrodynamic Interaction of Two Swimming Model Micro-Organisms." Journal of Fluid Mechanics, 568, 119.

Keulegan, G. and Carpenter, L. (1958). "Forces on cylinders and plates in an oscillating fluid." Journal of Research of the National Bureau of Standards, 60(5), 423.

Koch, D. L. and Subramanian, G. (2011). "Collective Hydrodynamics of Swimming Microorganisms: Living Fluids." Annual Review of Fluid Mechanics, 43(1), 637-659.

Lamb, H. (1932). Hydrodynamics. Cambridge University Press, Cambridge, 6th ed. edition.

Liao, J. C. (2003). "Fish Exploiting Vortices Decrease Muscle Activity." Science, 302(5650), $1566-1569$. 
Lin, Z., Liang, D., and Zhao, M. (2016). "Numerical Study of the Interaction between Two Immersed Cylinders." The 12th International Conference on Hydrodynamics, 55, <http://www.ichd2016.nl/onlineproc/proceedings/display_manuscript/55.htm>.

Lin, Z., Liang, D., and Zhao, M. (2017). "Interaction between Two Vibrating Cylinders Immersed in Fluid." Proceedings of the Twenty-seventh (2017) International Ocean and Polar Engineering Conference, number ISOPE-I-17-093, OnePetro, 1190, <https://www.onepetro.org/conference-paper/ISOPE-I-17-093>.

Lin, Z., Liang, D., and Zhao, M. (2018a). "Effects of Damping on Flow-Mediated Interaction Between Two Cylinders." Journal of Fluids Engineering, 140(9), 91106-91112.

Lin, Z., Liang, D., and Zhao, M. (2018b). "Flow-mediated interaction between a vibrating cylinder and an elastically-mounted cylinder." Ocean Engineering, 158, 389-402.

Lu, L., Liu, M.-m., Teng, B., Cui, Z.-d., Tang, G.-q., Zhao, M., and Cheng, L. (2014). "Numerical Investigation of Fluid Flow past Circular Cylinder with Multiple Control Rods at Low Reynolds Number." Journal of Fluids and Structures, 48, 235-259.

Metzger, B., Nicolas, M., and Guazzelli, É. (2007). "Falling Clouds of Particles in Viscous Fluids." Journal of Fluid Mechanics, 580, 283.

Morison, J., Johnson, J., and Schaaf, S. (1950). "The Force Exerted by Surface Waves on Piles." Journal of Petroleum Technology, 2(5), 149-154.

Nair, S. and Kanso, E. (2007). "Hydrodynamically Coupled Rigid Bodies." Journal of Fluid Mechanics, 592, 393-411. 
Riffell, J. a. and Zimmer, R. K. (2007). "Sex and Flow: the Consequences of Fluid Shear for Sperm-Egg Interactions." The Journal of experimental biology, 210(Pt 20), 3644-3660.

Sarpkaya, T. (1977). "In-line and transverse forces on smooth and sand-roughened cylinders in oscillatory flow at high reynolds numbers." Journal of Ship Research, 21(4), 200-216.

Tatsuno, M. and Bearman, P. W. (1990). "A Visual Study of the Flow around an Oscillating Circular Cylinder at Low Keulegan-Carpenter Numbers and Low Stokes Numbers." Journal of Fluid Mechanics, 211(-1), 157-182.

Tong, F., Cheng, L., Zhao, M., Zhou, T., and Chen, X. B. (2014). "The Vortex Shedding around Four Circular Cylinders in an In-Line Square Configuration." Physics of Fluids, $26(2), 024112$

Van Rees, W. M., Novati, G., and Koumoutsakos, P. (2015). "Self-Propulsion of a CounterRotating Cylinder Pair in a Viscous Fluid." Physics of Fluids, 27(6), 063102.

Voth, G. A., Bigger, B., Buckley, M. R., Losert, W., Brenner, M. P., Stone, H. A., Gollub, J. P., and Gollub, J. P. (2002). "Ordered Clusters and Dynamical States of Particles in a Vibrated Fluid." Physical Review Letters, 88(23), 2343011-2343014.

Weihs, D. (2004). "The Hydrodynamics of Dolphin Drafting." Journal of biology, 3(2), 8.1-8.16.

Williamson, C. H. K. (1985). "Sinusoidal Flow Relative to Circular Cylinders." Journal of Fluid Mechanics, 155, 141-174.

Zhao, M., Murphy, J. M., and Kwok, K. (2016). "Numerical Simulation of Vortex-Induced 
${ }_{682}$ Vibration of Two Rigidly Connected Cylinders in Side-By-Side and Tandem Arrangements 683 Using Rans Model." Journal of Fluids Engineering, 138(2), 21102. 


\section{List of Tables}

685 1 Comparison of the slave cylinder's displacement from different meshes for $G / D=0.9, A_{1} / D=0.477, f_{1} / f_{n}=3.2, m^{*}=1.5, \zeta=0, R e_{m}=10 . N_{c}$ is the element number along each cylinder's circumference, $\Delta r$ the minimum radial 


\begin{tabular}{lccccc}
\hline Mesh density & $N_{c}$ & $\Delta r$ & $N_{\text {node }}$ & $Y_{2, \min } / D$ & $Y_{2, \max } / D$ \\
Dense & 152 & 0.00130 & 30280 & -0.04893 & -0.1982 \\
Normal & 134 & 0.00144 & 24050 & -0.04804 & -0.1972 \\
Coarse & 86 & 0.00192 & 15971 & -0.04684 & -0.1955 \\
Very Coarse & 50 & 0.00597 & 9722 & -0.04463 & -0.1930 \\
\hline
\end{tabular}

TABLE 1: Comparison of the slave cylinder's displacement from different meshes for $G / D=0.9, A_{1} / D=0.477, f_{1} / f_{n}=3.2, m^{*}=1.5, \zeta=0, R e_{m}=$ 10. $N_{c}$ is the element number along each cylinder's circumference, $\Delta r$ the minimum radial mesh size and $N_{\text {node }}$ the total node number of the mesh. 


\section{List of Figures}

1 Tatsuno and Bearman's classification of flows [25]. They identified flow patterns within eight regimes indicated $A^{*}-G$, whereas the range of involved $K C$ and $\beta$ are indicated by solid circles. . . . . . . . . . . .

2 A sketch of interaction between two cylinders: While the master cylinder undergoes harmonic forced vibration, the slave cylinder is elastically mounted and vibrates passively along the y-axis. . . . . . . . . . .

3 Computational meshes for interaction between two cylinders $G / D=0.2$ with $\Delta r \leq 1.38 \times 10^{-3}$ and $N_{c} \geq 152 \ldots \ldots \ldots \ldots \ldots$

4 Comparison of the slave cylinder's displacement time histories from different meshes for $G / D=0.9, A_{1} / D=0.477, m^{*}=1.5, \zeta=0$ and (a) $R e_{m}=$ $10, f_{1} / f_{n}=3.2$, where the slave cylinder drifts towards the near side (b) $R e_{m}=150, f_{1} / f_{n}=3.2$, where the slave cylinder drifts towards the far side (c) $R e_{m}=150, f_{1} / f_{n}=0.725$, where the vibration amplitude is large due to the resonance. . . . . . . . . . . . . . . . . .

5 Amplitude spectra showing the responding displacement of the slave with $f_{1} / f_{n}=0.05-3.2$ at $G / D=0.9, A_{1} / D=0.477, m^{*}=1.5, \zeta=0$ with (a) $R e_{m}=10$ (b) $R e_{m}=30$ (c) $R e_{m}=50$ (d) $R e_{m}=110$. The dashed thin line tracks the dominant frequencies. 
6 (a) Variation of amplification factor $A_{2} / A_{1}$ with master cylinder's oscillation frequency $f_{1} / f_{n}$ (b) Zoom-in at primary and secondary resonance regimes and (c) Variation of slave-master phase difference $\Delta \phi_{21}$ with $f_{1} / f_{n}$ (for the frequency components with $\left.f / f_{n}=f_{1} / f_{n}\right)$ with $f_{1} / f_{n}$ at $G / D=0.9, A_{1} / D=$ $0.477, m^{*}=1.5, \zeta=0, R e_{m}=10-150$, and $K C=3, \beta=3.3-50 . A_{2} / A_{1}$ is positively correlated with $R e_{m}$, particularly within the regime of resonance, and the resonance frequency increases with $R e_{m}$. The resonance amplitude at $R e_{m}=150$ is as large as 1.6 times of that at $R e_{m}=10$. The phase difference is shifted towards the positive side with the increase of $R e_{m}$.

7 (a) Variation of slave cylinder's amplification factor $A_{2} / A_{1}$ with master cylinder's oscillation frequency $f_{1} / f_{n}$ at $G / D=0.9, A_{1} / D=0.477, m^{*}=2.0, R e_{m}=$ $10-110$ and $\zeta=0-0.2$. (b) Zoom-in at resonance regimes. The marker type denotes damping factor $\zeta$, whereas the line type denotes the Reynolds number $R e_{m} \ldots \ldots \ldots \ldots \ldots \ldots \ldots \ldots \ldots \ldots \ldots \ldots \ldots$

8 (a) Variation of slave cylinder's amplification factor $A_{2} / A_{1}$ with master cylinder's oscillation frequency $f_{1} / f_{n}$ at $G / D=0.9, A_{1} / D=0.477, \zeta=0, m^{*}=$ $1.5-2.5, R e_{m}=10-110$ and (b) Zoom-in at resonance regimes. The marker type denotes mass ratio $m^{*}$, whereas the line type denotes the Reynolds num-

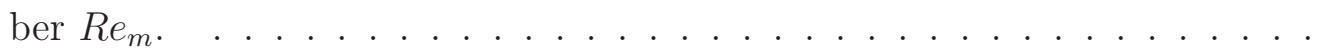

9 (a) Variation in vibration centre drift $\Delta \bar{Y}_{2}$ with $f_{1} / f_{n}$ at $G / D=0.9, A_{1} / D=$ $0.477, m^{*}=1.5, \zeta=0$ and $R e_{m}=10-150$. (b) Zoom-in at the secondary and the primary resonance regime (c) Further zoom-in at the primary resonance regime (d) Variation in vibration centre drift $\Delta \bar{Y}_{2}$ with $R e_{m}$ at $G / D=0.9, A_{1} / D=0.477, m^{*}=1.5, \zeta=0$ and $f_{1} / f_{n}=1-3.2 . \ldots$.

10 (a) Variation in $\Delta \bar{Y}_{2} / A_{1}$ with $f_{1} / f_{n}$ and (b) Zoom-in for its resonance regime at $G / D=0.9, A_{1} / D=0.477, m^{*}=1.5, R e_{m}=10,70$ and $\zeta=0-0.2$. The marker type denotes $\zeta$, whereas the line type denotes $R e_{m} \ldots \ldots$ 
11 (a) Variation in $\Delta \bar{Y}_{2} / A_{1}$ with $f_{1} / f_{n}$ and (b) Zoom-in for its resonance regime at $G / D=0.9, A_{1} / D=0.477, \zeta=0.2, R e_{m}=10,70$ and $m^{*}=1.5-2.5$. The marker type denotes $m^{*}$, whereas the line type denotes $R e_{m} \ldots \ldots \ldots$

12 Contours of pressure coefficient $C_{p}$ and velocity vectors (1) in the gap at $\phi_{1}=180^{\circ}$ and (2) in the far side of the master cylinder at $\phi_{1}=0^{\circ}$, given $G / D=0.9, A_{1} / D=0.477, m^{*}=1.5, f_{1} / f_{n}=2.8$, with (a) $R e_{m}=10, \Delta \phi_{21}=$ $-30.6^{\circ}$; (b) $R e_{m}=50, \Delta \phi_{21}=-16.8^{\circ}$; (c) $R e_{m}=110, \Delta \phi_{21}=-12.22^{\circ}$. The velocity vector is drawn on every two grid points and the vector scale factors are 0.1 grid units/magnitude.

13 Pressure coefficient contours and velocity vectors in the gap at $G / D=0.9, A_{1} / D=$ $0.477, m^{*}=1.5, \zeta=0$, and $\phi_{1}=180^{\circ}$ with (a) $R e_{m}=10, f_{1} / f_{n}=0.65, \Delta \phi_{21}=$ $27.05^{\circ}$, (b) $R e_{m}=50, f_{1} / f_{n}=0.7, \Delta \phi_{21}=64.66^{\circ}$, (c) $R e_{m}=110, f_{1} / f_{n}=$ $0.72, \Delta \phi_{21}=71.42^{\circ}$, The velocity vector is drawn on every two grid points and the vector scale factors are 0.1 grid units/magnitude. . . . . . . . .

14 Pressure coefficient contours and streamlines at $G / D=0.9, A_{1} / D=0.477, m^{*}=$ $1.5, \zeta=0$, and (1) $R e_{m}=10, f_{1} / f_{n}=2.8, \Delta \phi_{21}=-30.64^{\circ}$, (2) $R e_{m}=$ $50, f_{1} / f_{n}=2.8, \Delta \phi_{21}=-16.77^{\circ}$, (3) $R e_{m}=110, f_{1} / f_{n}=2.8, \Delta \phi_{21}=$ $-12.22^{\circ}$, (a) $\phi_{1}=0^{\circ}$ (b) $\phi_{1}=90^{\circ}$ (c) $\phi_{1}=180^{\circ}$ (d) $\phi_{1}=270^{\circ}$. The black sticks indicate the phases of the cylinders.

15 Evolution of vorticity contours at $G / D=0.9, A_{1} / D=0.477, m^{*}=1.5, \zeta=$ $0, f_{1} / f_{n}=3.2$, and (1) $R e_{m}=10$, (2) $R e_{m}=150$, (a) $\phi_{1}=0^{\circ}$ (b) $\phi_{1}=90^{\circ}$ (c) $\phi_{1}=180^{\circ}$ (d) $\phi_{1}=270^{\circ}$.

16 Streamlines and vorticity contours at $G / D=0.9, A_{1} / D=0.477, m^{*}=1.5, \zeta=$ $0, f_{1} / f_{n}=3.2, \phi_{1}=180^{\circ}$, and (a) $R e_{m}=10$, (b) $R e_{m}=150$. 
17 Time histories of hydraulic force upon the slave cylinder at primary or secondary resonance with $G / D=0.9, A_{1} / D=0.477, m^{*}=2$, and (a) $\zeta=0$, $R e_{m}=10, f_{1} / f_{n}=0.7$ (b) $\zeta=0, R e_{m}=50, f_{1} / f_{n}=0.745$ (c) $\zeta=0$, $R e_{m}=110, f_{1} / f_{n}=0.765$ (d) $\zeta=0.2, R e_{m}=10, f_{1} / f_{n}=0.73$ (e) $\zeta=0.2$, $R e_{m}=50, f_{1} / f_{n}=0.775$ (f) $\zeta=0.2, R e_{m}=110, f_{1} / f_{n}=0.795(\mathrm{~g}) \zeta=0$, $R e_{m}=10, f_{1} / f_{n}=0.39$ (h) $\zeta=0, R e_{m}=50, f_{1} / f_{n}=0.39$ (i) $\zeta=0$, $R e_{m}=110, f_{1} / f_{n}=0.39(\mathrm{j}) \zeta=0.2, R e_{m}=10, f_{1} / f_{n}=0.39(\mathrm{k}) \zeta=0.2$, $R e_{m}=50, f_{1} / f_{n}=0.39$ (l) $\zeta=0.2, R e_{m}=110, f_{1} / f_{n}=0.39 \ldots$

18 Pressure coefficient contours and velocity vectors at $G / D=0.9, A_{1} / D=$ $0.477, m^{*}=2, \zeta=0$ and (1) $R e_{m}=10, f_{1} / f_{n}=0.7$, (2) $R e_{m}=50, f_{1} / f_{n}=$ $0.745,(3) R e_{m}=110, f_{1} / f_{n}=0.765$, (a) $\phi_{1}=0^{\circ}$ (b) $\phi_{1}=90^{\circ}$ (c) $\phi_{1}=180^{\circ}$ (d) $\phi_{1}=270^{\circ}$. Primary resonance occurs at around $f_{1} / f_{n}=0.75$ and the damping factor is zero. . . . . . . . . . . . . . . .

19 Pressure coefficient contours and velocity vectors at $G / D=0.9, A_{1} / D=$ $0.477, m^{*}=2, \zeta=0.2$ and (1) $R e_{m}=10, f_{1} / f_{n}=0.73$, (2) $R e_{m}=50, f_{1} / f_{n}=$ 0.775, (3) $R e_{m}=110, f_{1} / f_{n}=0.795$, (a) $\phi_{1}=0^{\circ}$ (b) $\phi_{1}=90^{\circ}$ (c) $\phi_{1}=180^{\circ}$ (d) $\phi_{1}=270^{\circ}$. Primary resonance occurs at around $f_{1} / f_{n}=0.75$ and the damping factor is relatively high at $\zeta=0.2$.

20 Pressure coefficient contours and velocity vectors at $G / D=0.9, A_{1} / D=$ 0.477, $m^{*}=2, \zeta=0, f_{1} / f_{n}=0.39$ and (1) $R e_{m}=10$, (2) $R e_{m}=50$, (3) $R e_{m}=110$, (a) $\phi_{1}=0^{\circ}$ (b) $\phi_{1}=90^{\circ}$ (c) $\phi_{1}=180^{\circ}$ (d) $\phi_{1}=270^{\circ}$. Secondary resonance occurs at $f_{1} / f_{n}=0.39$ and the damping factor is zero. . . . . .

21 Pressure coefficient contours and velocity vectors at $G / D=0.9, A_{1} / D=$ $0.477, m^{*}=2, \zeta=0.2, f_{1} / f_{n}=0.39$ and (1) $R e_{m}=10$, (2) $R e_{m}=50$, (3) $R e_{m}=110$, (a) $\phi_{1}=0^{\circ}$ (b) $\phi_{1}=90^{\circ}$ (c) $\phi_{1}=180^{\circ}$ (d) $\phi_{1}=270^{\circ}$. Secondary resonance occurs at $f_{1} / f_{n}=0.39$ and the damping factor is relatively high at $\zeta=0.2$. 


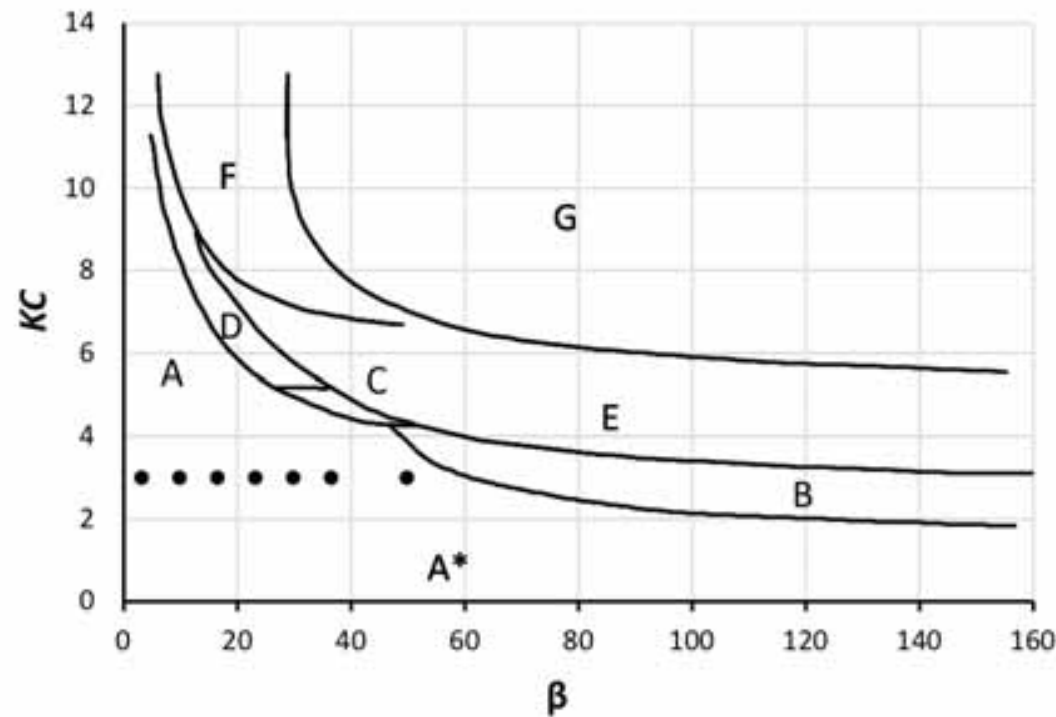

FIG. 1: Tatsuno and Bearman's classification of flows [25]. They identified flow patterns within eight regimes indicated $A^{*}-G$, whereas the range of involved $K C$ and $\beta$ are indicated by solid circles. 


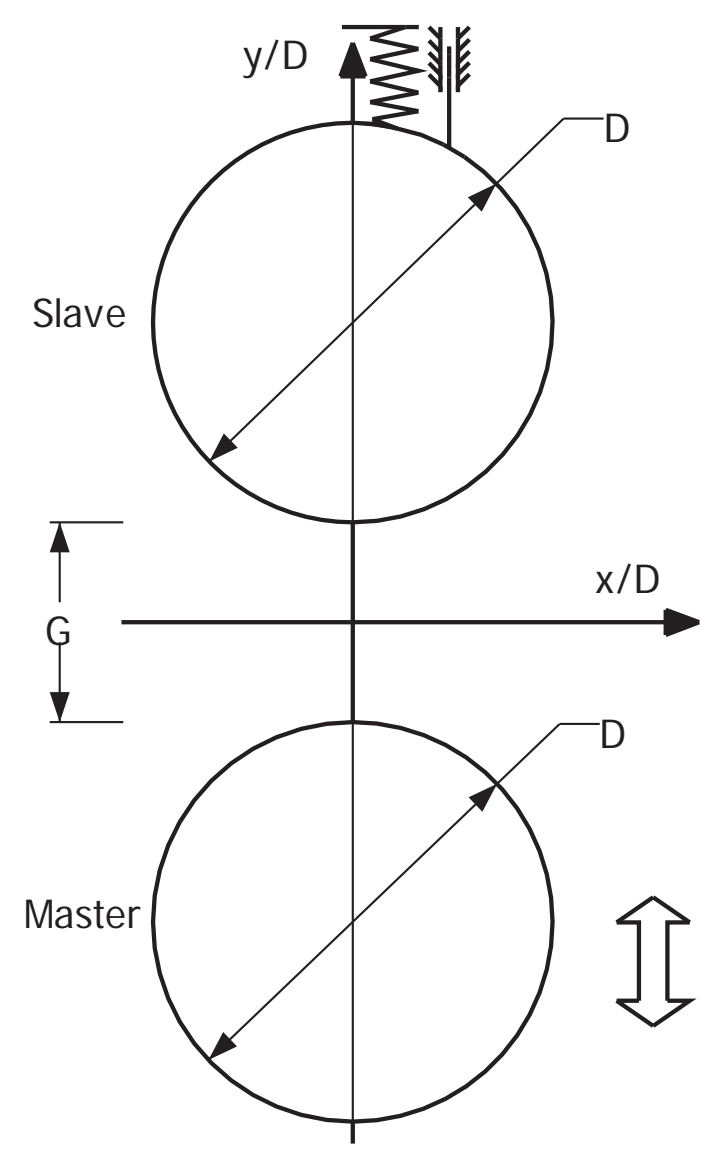

FIG. 2: A sketch of interaction between two cylinders: While the master cylinder undergoes harmonic forced vibration, the slave cylinder is elastically mounted and vibrates passively along the y-axis. 


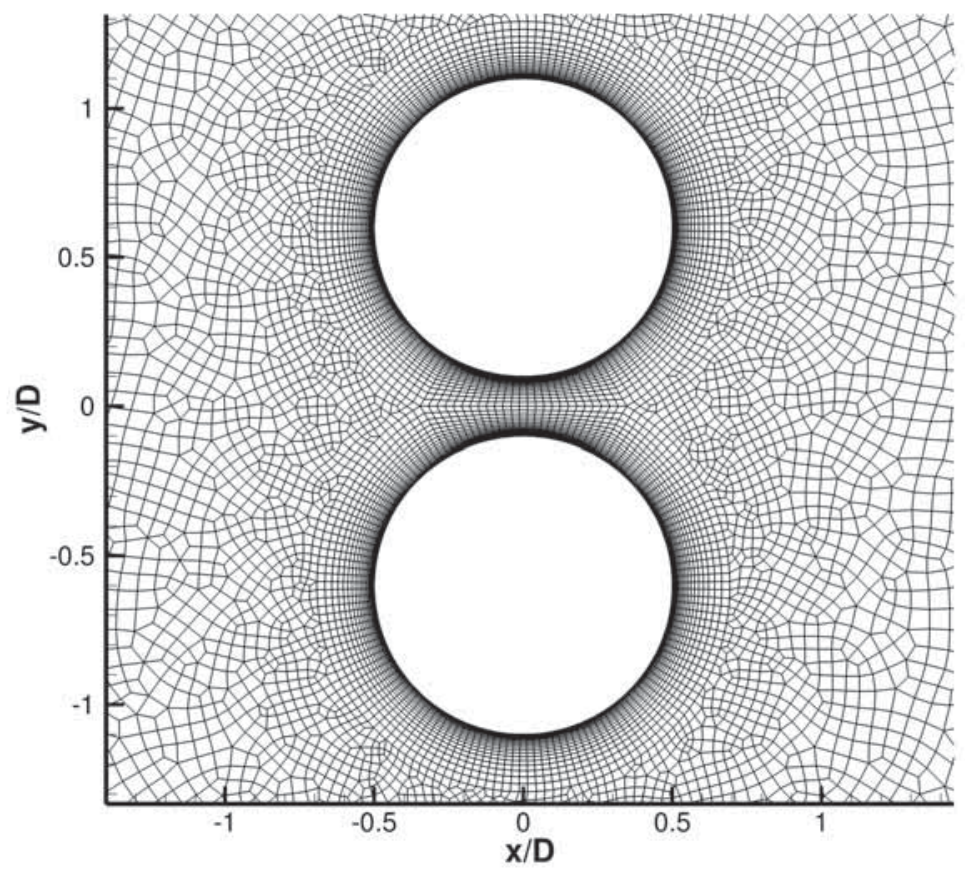

FIG. 3: Computational meshes for interaction between two cylinders $G / D=$ 0.2 with $\Delta r \leq 1.38 \times 10^{-3}$ and $N_{c} \geq 152$ 

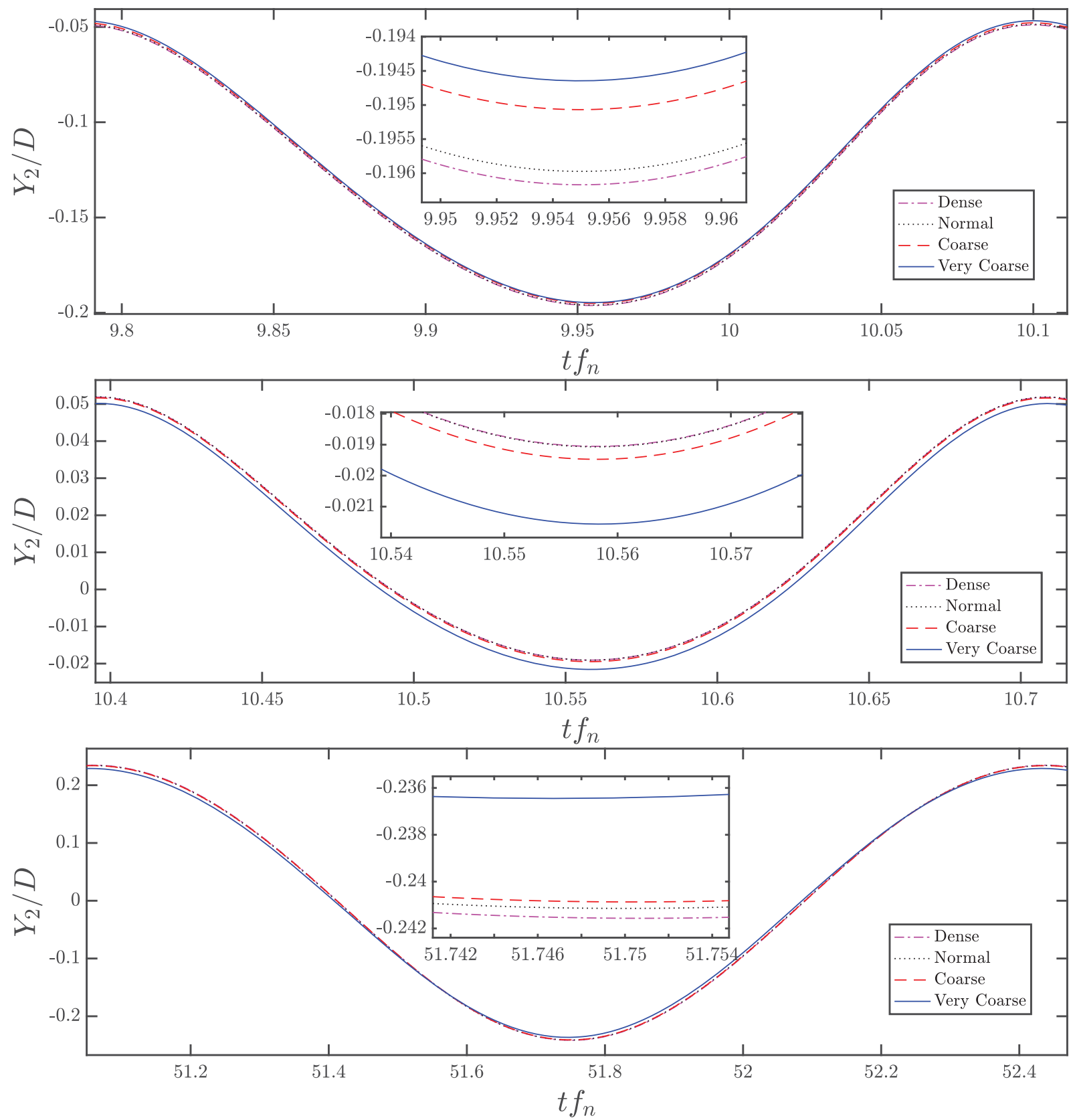

FIG. 4: Comparison of the slave cylinder's displacement time histories from different meshes for $G / D=0.9, A_{1} / D=0.477, m^{*}=1.5, \zeta=0$ and (a) $R e_{m}=10, f_{1} / f_{n}=3.2$, where the slave cylinder drifts towards the near side (b) $R e_{m}=150, f_{1} / f_{n}=3.2$, where the slave cylinder drifts towards the far side (c) $R e_{m}=150, f_{1} / f_{n}=0.725$, where the vibration amplitude is large due to the resonance. 

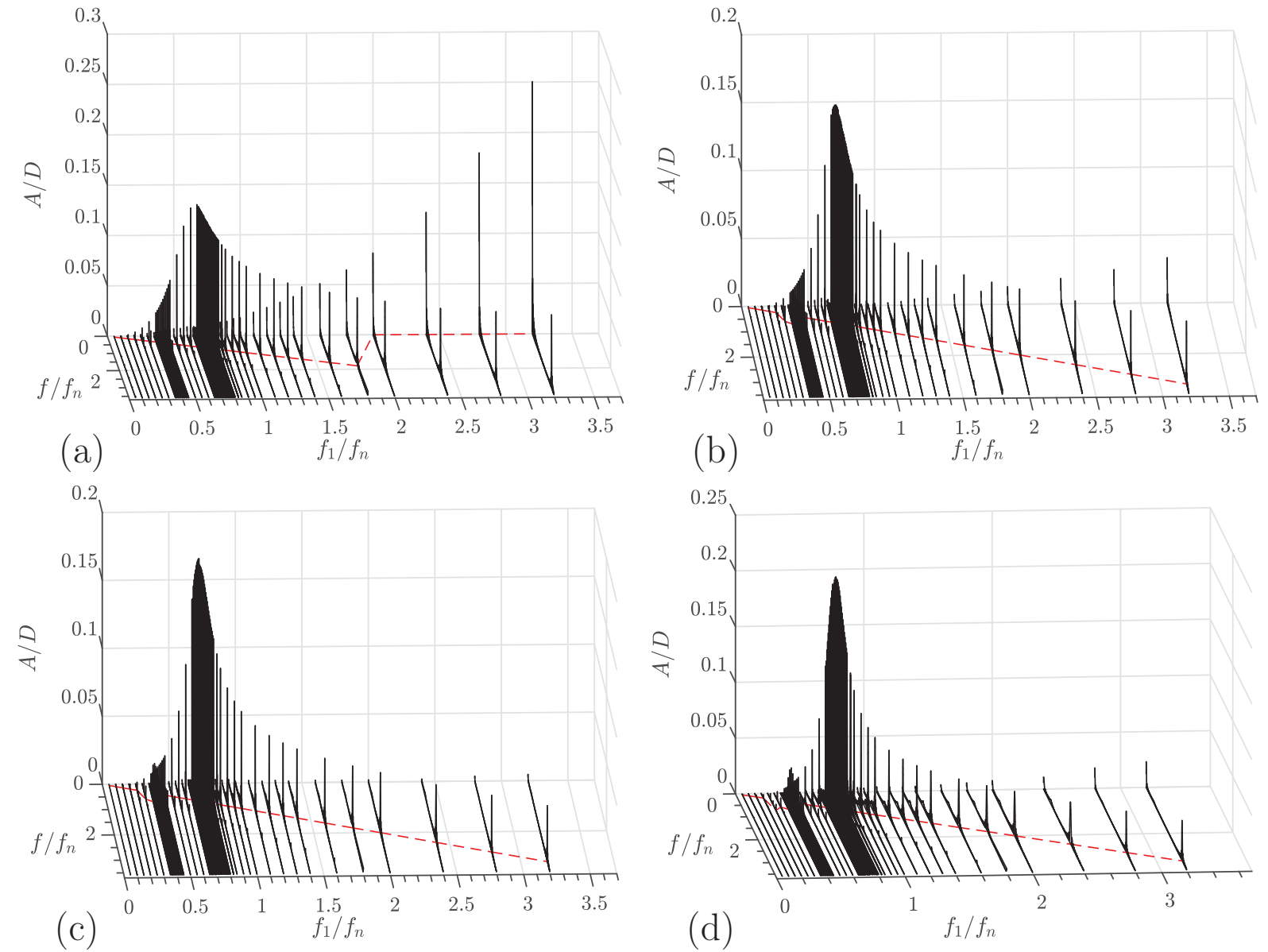

FIG. 5: Amplitude spectra showing the responding displacement of the slave with $f_{1} / f_{n}=0.05-3.2$ at $G / D=0.9, A_{1} / D=0.477, m^{*}=1.5, \zeta=0$ with (a) $R e_{m}=10$ (b) $R e_{m}=30$ (c) $R e_{m}=50$ (d) $R e_{m}=110$. The dashed thin line tracks the dominant frequencies. 

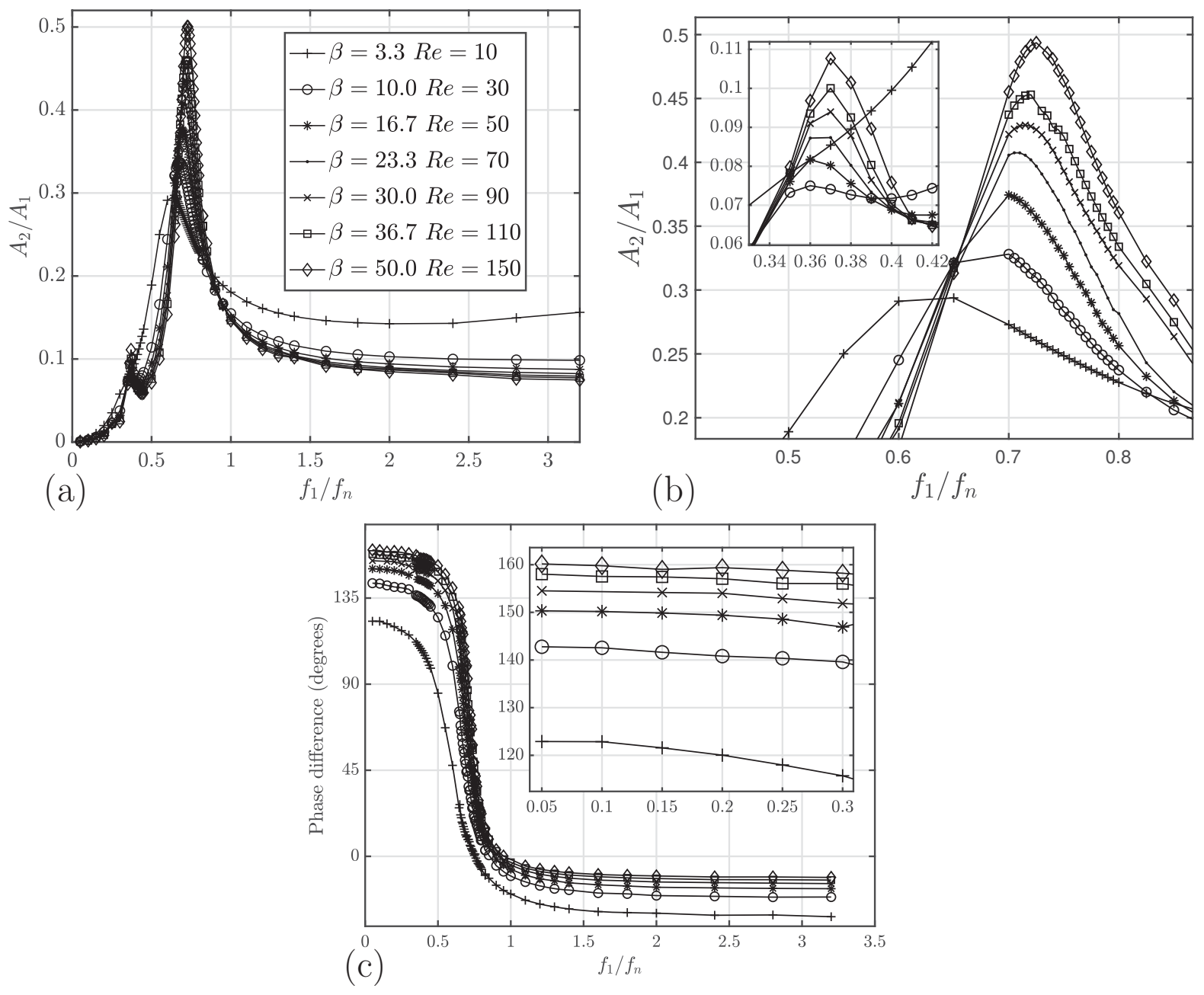

FIG. 6: (a) Variation of amplification factor $A_{2} / A_{1}$ with master cylinder's oscillation frequency $f_{1} / f_{n}$ (b) Zoom-in at primary and secondary resonance regimes and (c) Variation of slave-master phase difference $\Delta \phi_{21}$ with $f_{1} / f_{n}$ (for the frequency components with $f / f_{n}=f_{1} / f_{n}$ ) with $f_{1} / f_{n}$ at $G / D=$ $0.9, A_{1} / D=0.477, m^{*}=1.5, \zeta=0, R_{m}=10-150$, and $K C=3, \beta=$ 3.3-50. $A_{2} / A_{1}$ is positively correlated with $R e_{m}$, particularly within the regime of resonance, and the resonance frequency increases with $R e_{m}$. The resonance amplitude at $R e_{m}=150$ is as large as 1.6 times of that at $R e_{m}=10$. The phase difference is shifted towards the positive side with the increase of $R e_{m}$. 

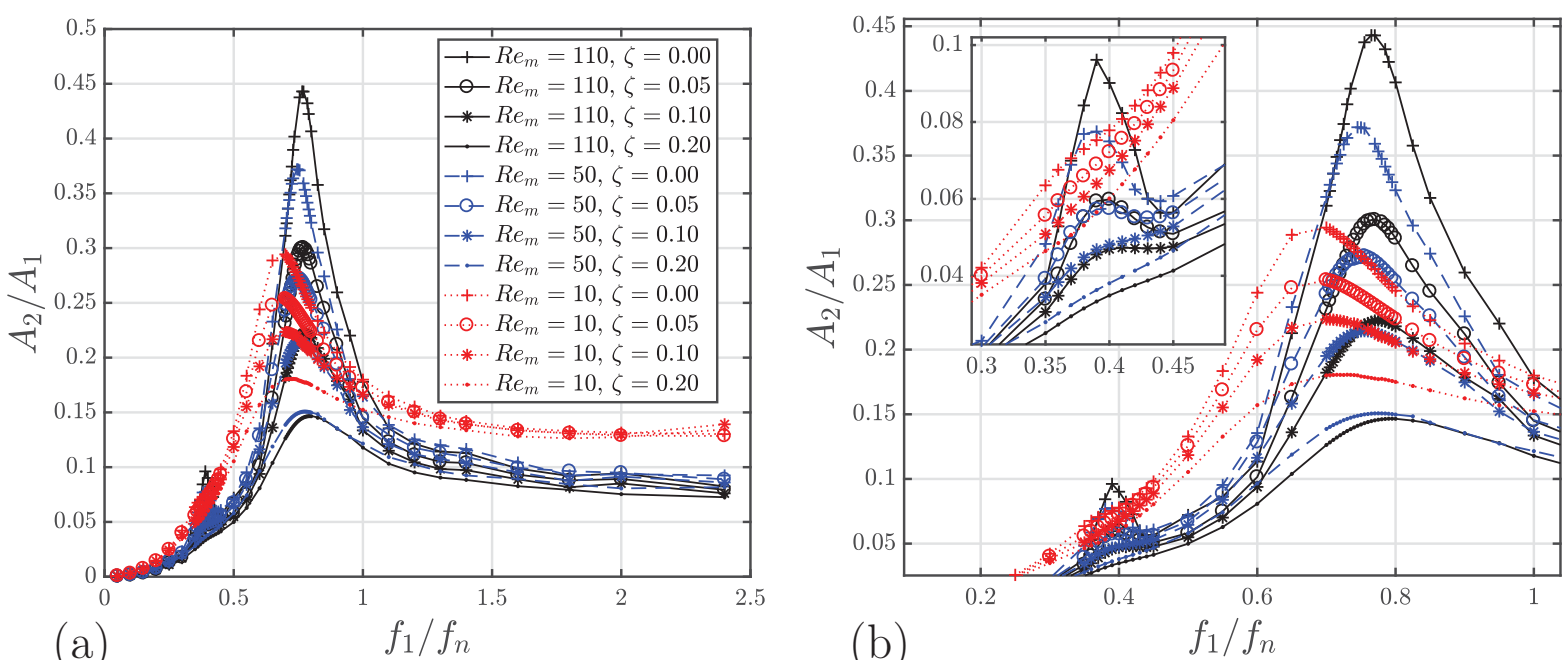

FIG. 7: (a) Variation of slave cylinder's amplification factor $A_{2} / A_{1}$ with master cylinder's oscillation frequency $f_{1} / f_{n}$ at $G / D=0.9, A_{1} / D=0.477, m^{*}=$ $2.0, R e_{m}=10-110$ and $\zeta=0-0.2$. (b) Zoom-in at resonance regimes. The marker type denotes damping factor $\zeta$, whereas the line type denotes the Reynolds number $R e_{m}$. 

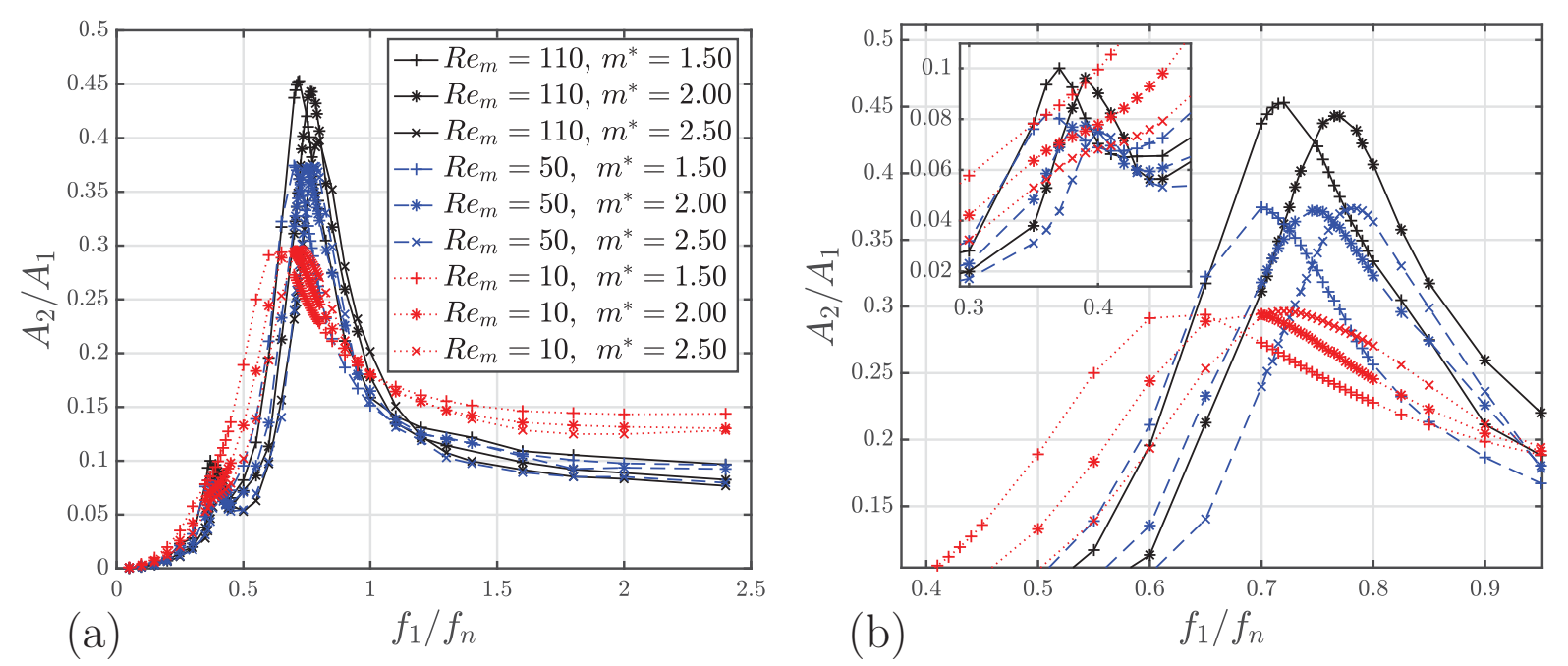

FIG. 8: (a) Variation of slave cylinder's amplification factor $A_{2} / A_{1}$ with master cylinder's oscillation frequency $f_{1} / f_{n}$ at $G / D=0.9, A_{1} / D=0.477, \zeta=$ $0, m^{*}=1.5-2.5, R e_{m}=10-110$ and (b) Zoom-in at resonance regimes. The marker type denotes mass ratio $m^{*}$, whereas the line type denotes the Reynolds number $R e_{m}$. 


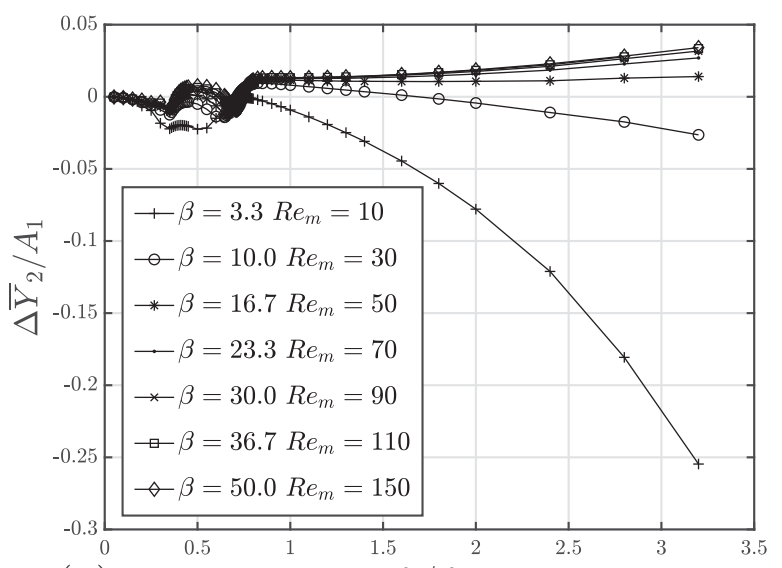

(a)

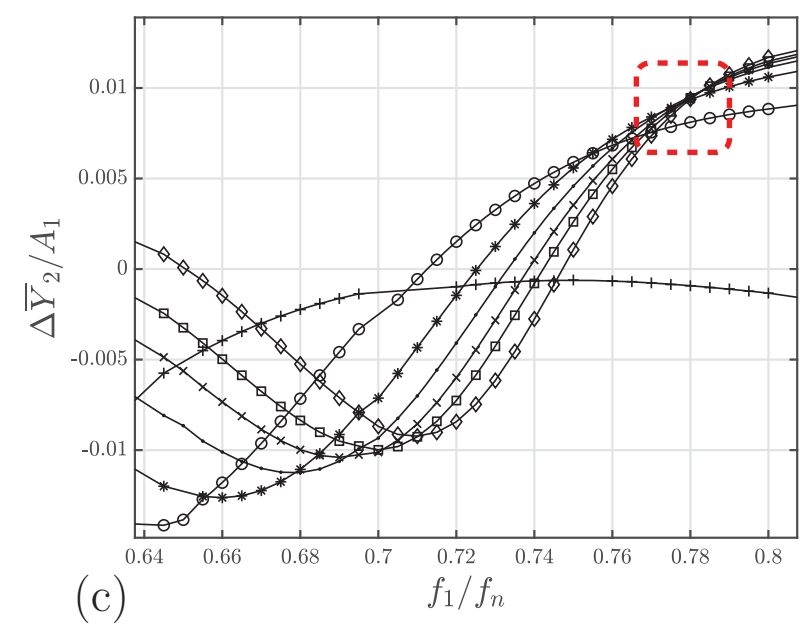

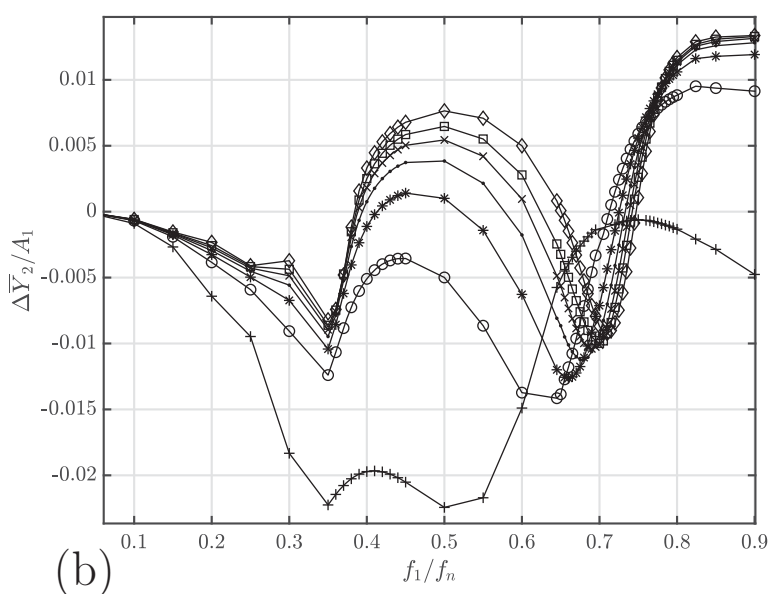

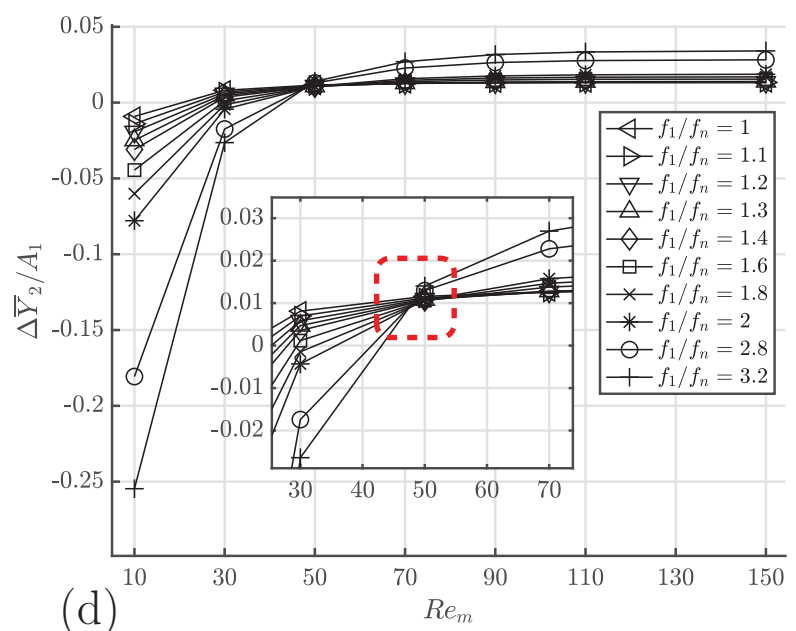

FIG. 9: (a) Variation in vibration centre drift $\Delta \bar{Y}_{2}$ with $f_{1} / f_{n}$ at $G / D=$ $0.9, A_{1} / D=0.477, m^{*}=1.5, \zeta=0$ and $R e_{m}=10-150$. (b) Zoom-in at the secondary and the primary resonance regime (c) Further zoom-in at the primary resonance regime (d) Variation in vibration centre drift $\Delta \bar{Y}_{2}$ with $R e_{m}$ at $G / D=0.9, A_{1} / D=0.477, m^{*}=1.5, \zeta=0$ and $f_{1} / f_{n}=1-3.2$. 

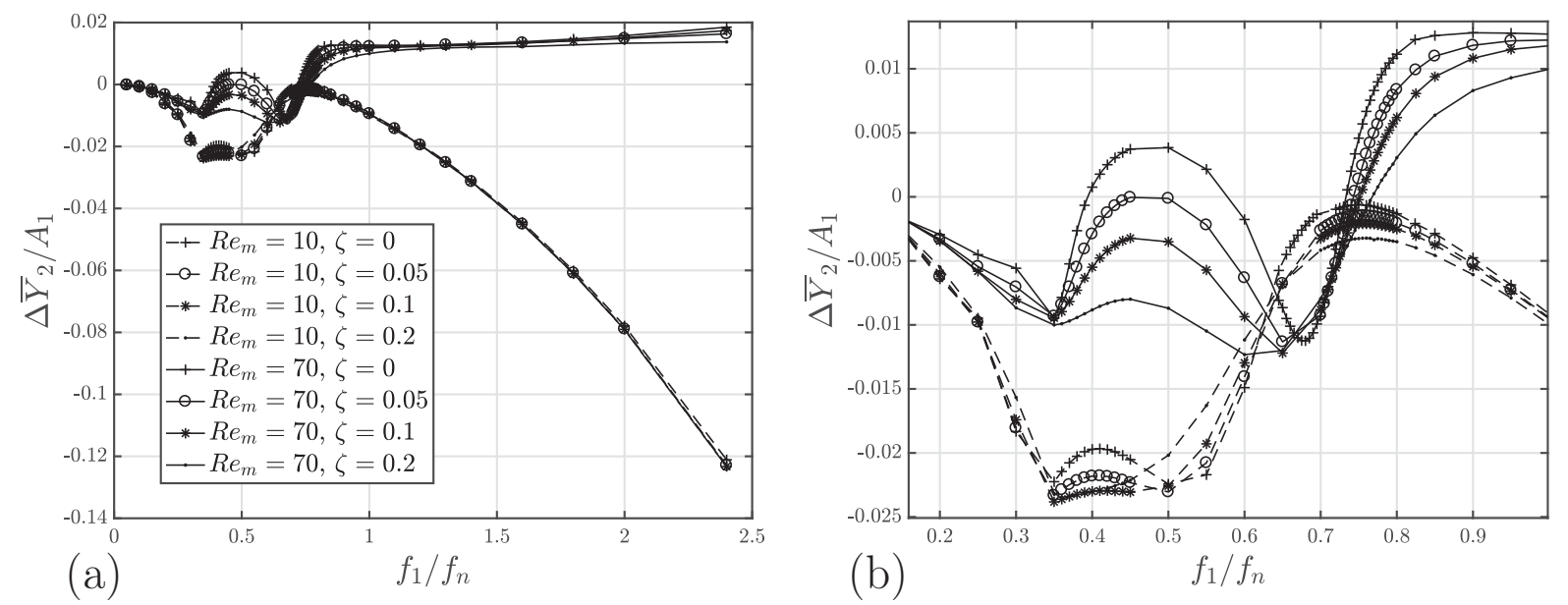

FIG. 10: (a) Variation in $\Delta \bar{Y}_{2} / A_{1}$ with $f_{1} / f_{n}$ and (b) Zoom-in for its resonance regime at $G / D=0.9, A_{1} / D=0.477, m^{*}=1.5, R e_{m}=10,70$ and $\zeta=0-0.2$. The marker type denotes $\zeta$, whereas the line type denotes $R e_{m}$. 


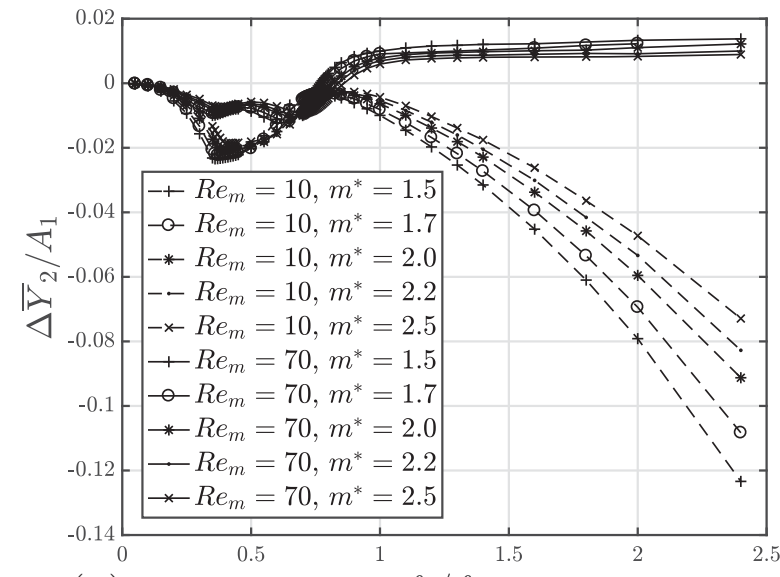

(a) $f_{1} / f_{n}$

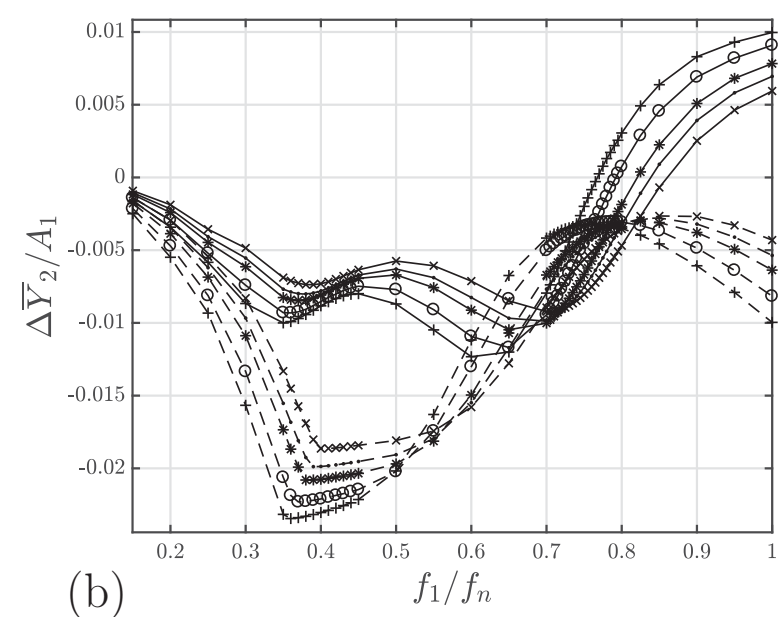

(b)

FIG. 11: (a) Variation in $\Delta \bar{Y}_{2} / A_{1}$ with $f_{1} / f_{n}$ and (b) Zoom-in for its resonance regime at $G / D=0.9, A_{1} / D=0.477, \zeta=0.2, R e_{m}=10,70$ and $m^{*}=1.5-$ 2.5. The marker type denotes $m^{*}$, whereas the line type denotes $R e_{m}$. 

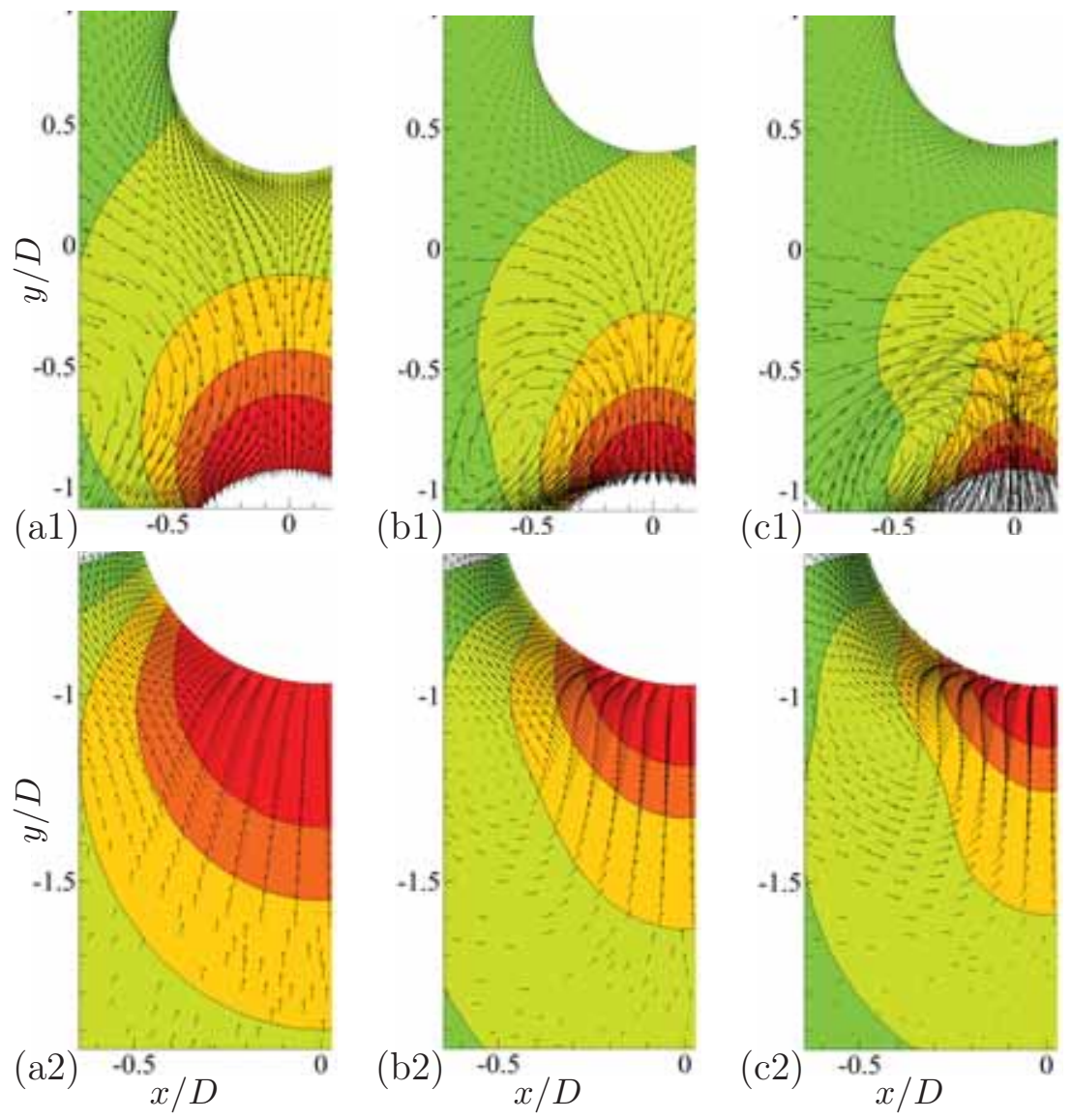

FIG. 12: Contours of pressure coefficient $C_{p}$ and velocity vectors (1) in the gap at $\phi_{1}=180^{\circ}$ and (2) in the far side of the master cylinder at $\phi_{1}=0^{\circ}$, given $G / D=0.9, A_{1} / D=0.477, m^{*}=1.5, f_{1} / f_{n}=2.8$, with (a) $R e_{m}=10, \Delta \phi_{21}=$ $-30.6^{\circ}$; (b) $R e_{m}=50, \Delta \phi_{21}=-16.8^{\circ}$; (c) $R e_{m}=110, \Delta \phi_{21}=-12.22^{\circ}$. The velocity vector is drawn on every two grid points and the vector scale factors are 0.1 grid units/magnitude. 

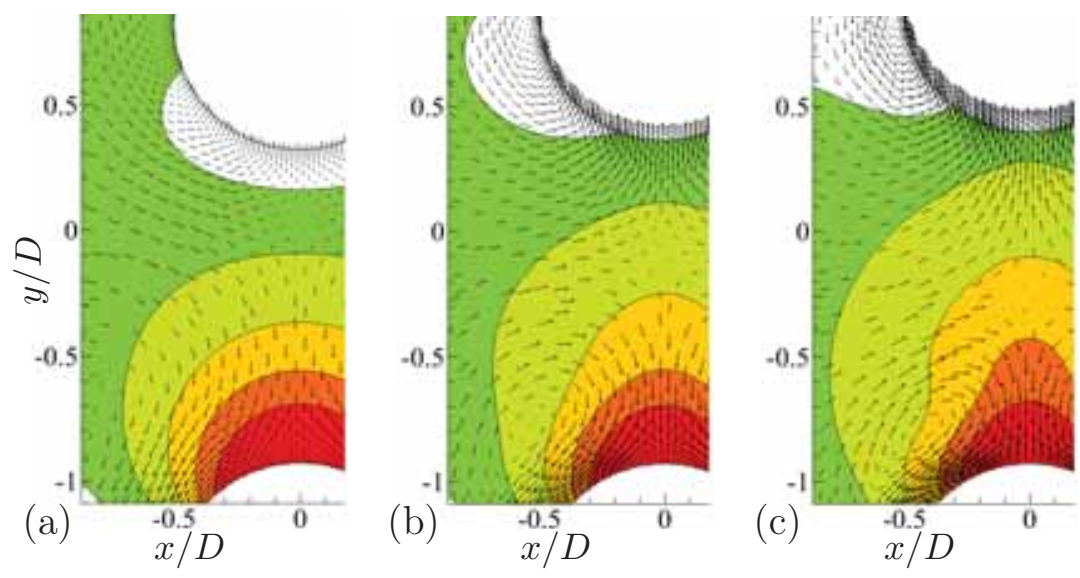

FIG. 13: Pressure coefficient contours and velocity vectors in the gap at $G / D=0.9, A_{1} / D=0.477, m^{*}=1.5, \zeta=0$, and $\phi_{1}=180^{\circ}$ with (a) $R e_{m}=$ $10, f_{1} / f_{n}=0.65, \Delta \phi_{21}=27.05^{\circ}$, (b) $R e_{m}=50, f_{1} / f_{n}=0.7, \Delta \phi_{21}=64.66^{\circ}$, (c) $R e_{m}=110, f_{1} / f_{n}=0.72, \Delta \phi_{21}=71.42^{\circ}$, The velocity vector is drawn on every two grid points and the vector scale factors are 0.1 grid units/magnitude. 

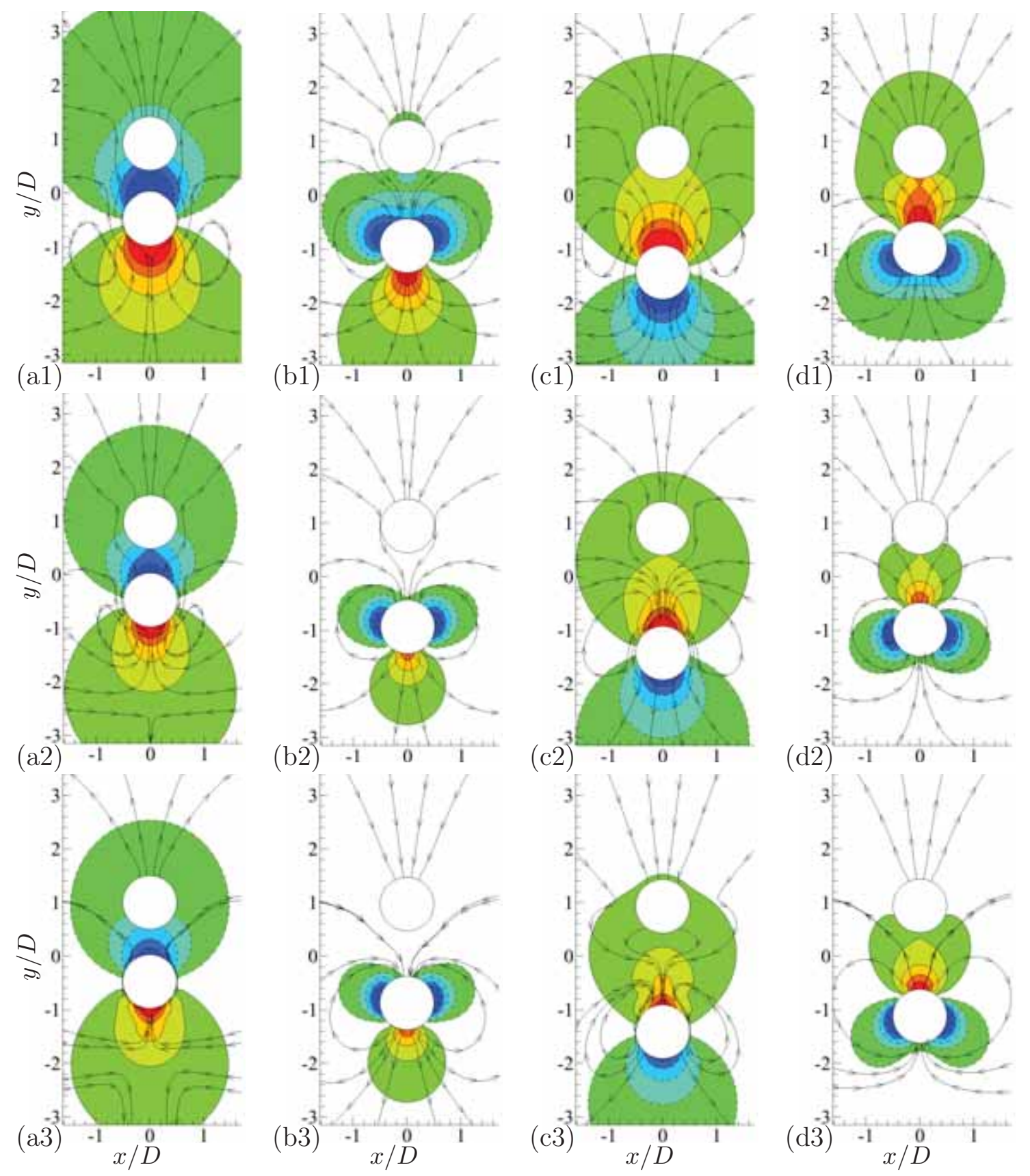

FIG. 14: Pressure coefficient contours and streamlines at $G / D=0.9, A_{1} / D=$ $0.477, m^{*}=1.5, \zeta=0$, and (1) $R e_{m}=10, f_{1} / f_{n}=2.8, \Delta \phi_{21}=-30.64^{\circ}$, (2) $R e_{m}=50, f_{1} / f_{n}=2.8, \Delta \phi_{21}=-16.77^{\circ}$, (3) $R e_{m}=110, f_{1} / f_{n}=2.8, \Delta \phi_{21}=$ $-12.22^{\circ}$, (a) $\phi_{1}=0^{\circ}$ (b) $\phi_{1}=90^{\circ}$ (c) $\phi_{1}=180^{\circ}$ (d) $\phi_{1}=270^{\circ}$. The black sticks indicate the phases of the cylinders. 

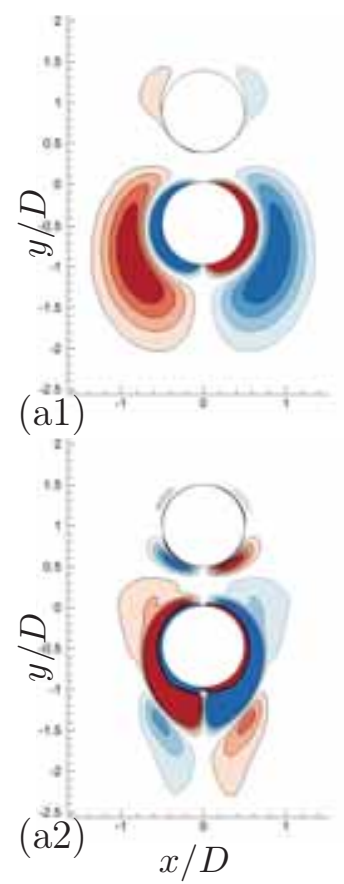
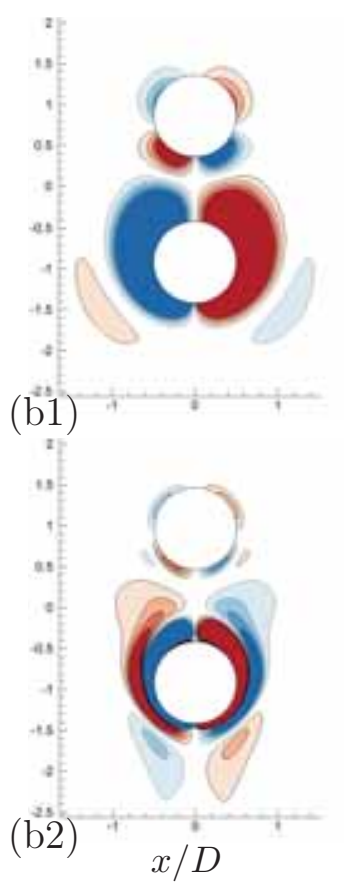
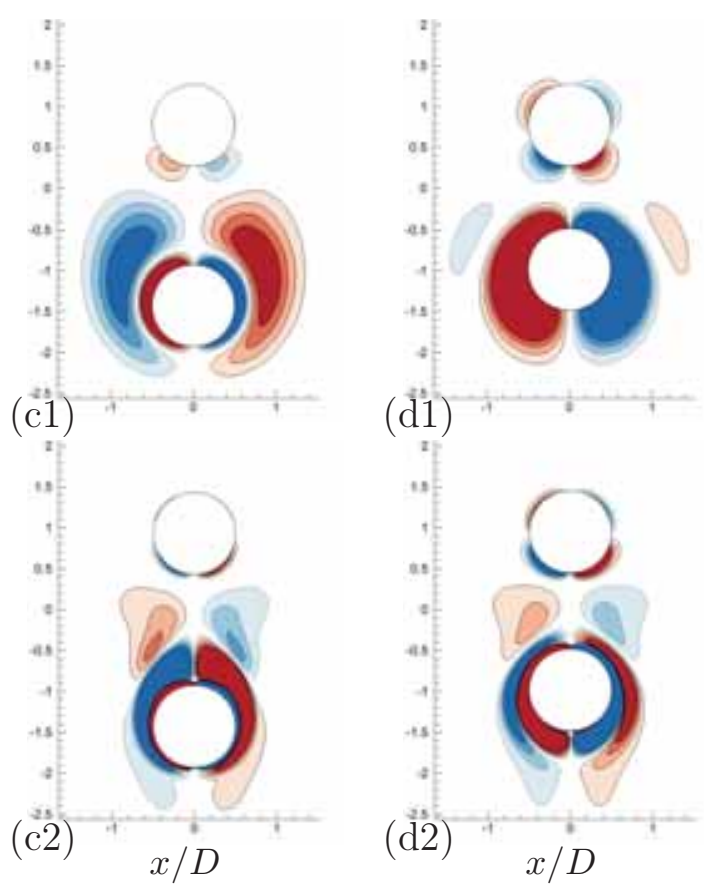

FIG. 15: Evolution of vorticity contours at $G / D=0.9, A_{1} / D=0.477, m^{*}=$ $1.5, \zeta=0, f_{1} / f_{n}=3.2$, and (1) $R e_{m}=10$, (2) $R e_{m}=150$, (a) $\phi_{1}=0^{\circ}$ (b) $\phi_{1}=90^{\circ}$ (c) $\phi_{1}=180^{\circ}$ (d) $\phi_{1}=270^{\circ}$. 

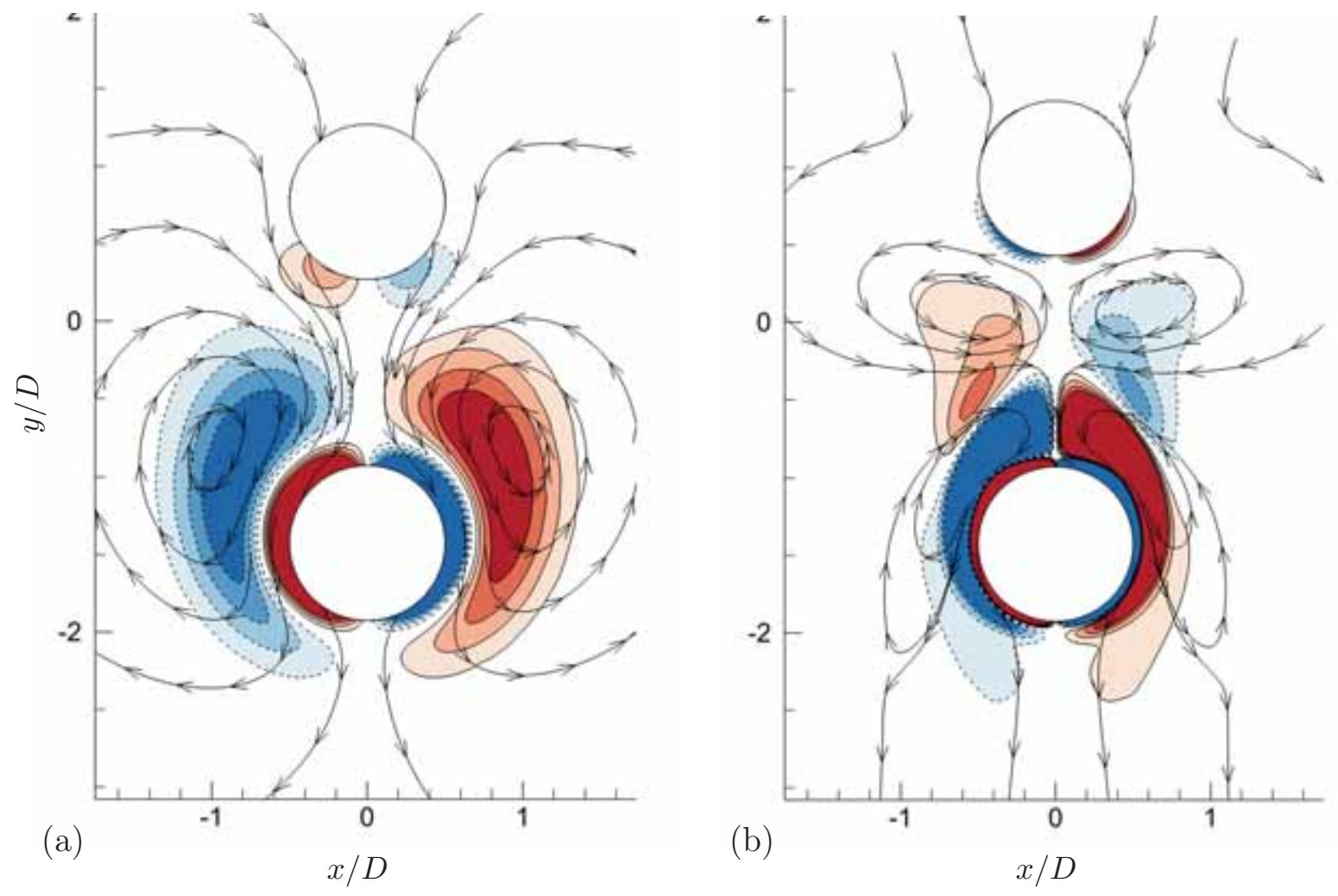

FIG. 16: Streamlines and vorticity contours at $G / D=0.9, A_{1} / D=$ $0.477, m^{*}=1.5, \zeta=0, f_{1} / f_{n}=3.2, \phi_{1}=180^{\circ}$, and (a) $R e_{m}=10$, (b) $R e_{m}=150$. 

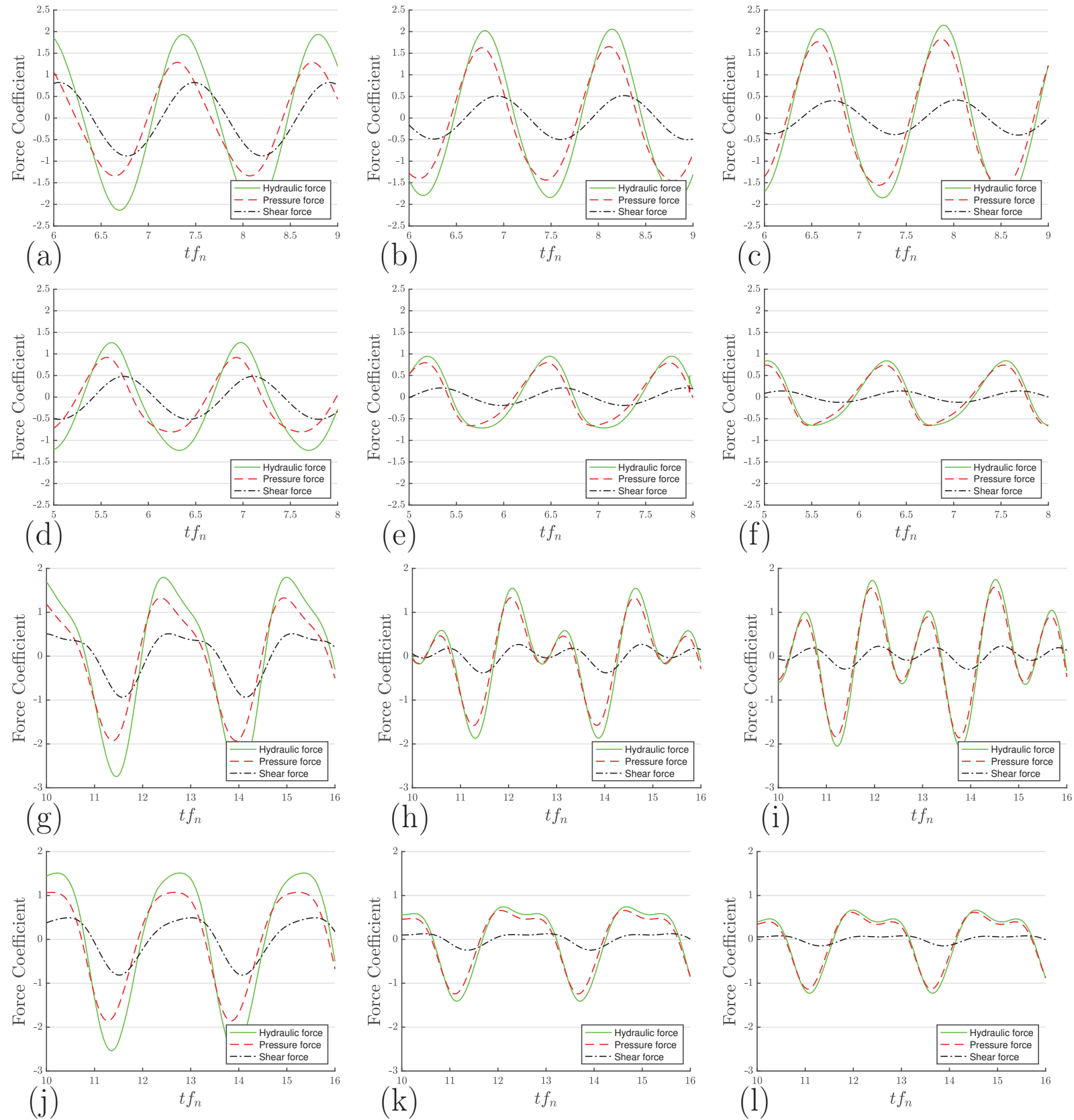

FIG. 17: Time histories of hydraulic force upon the slave cylinder at primary or secondary resonance with $G / D=0.9, A_{1} / D=0.477, m^{*}=2$, and (a) $\zeta=0$, $R e_{m}=10, f_{1} / f_{n}=0.7$ (b) $\zeta=0, R e_{m}=50, f_{1} / f_{n}=0.745$ (c) $\zeta=0$, $R e_{m}=110, f_{1} / f_{n}=0.765$ (d) $\zeta=0.2, R e_{m}=10, f_{1} / f_{n}=0.73$ (e) $\zeta=0.2$, $R e_{m}=50, f_{1} / f_{n}=0.775$ (f) $\zeta=0.2, R e_{m}=110, f_{1} / f_{n}=0.795(\mathrm{~g}) \zeta=0$, $R e_{m}=10, f_{1} / f_{n}=0.39$ (h) $\zeta=0, R e_{m}=50, f_{1} / f_{n}=0.39$ (i) $\zeta=0$, $R e_{m}=110, f_{1} / f_{n}=0.39(\mathrm{j}) \zeta=0.2, R e_{m}=10, f_{1} / f_{n}=0.39(\mathrm{k}) \zeta=0.2$, $R e_{m}=50, f_{1} / f_{n}=0.39$ (l) $\zeta=0.2, R e_{m}=110, f_{1} / f_{n}=0.39$ 

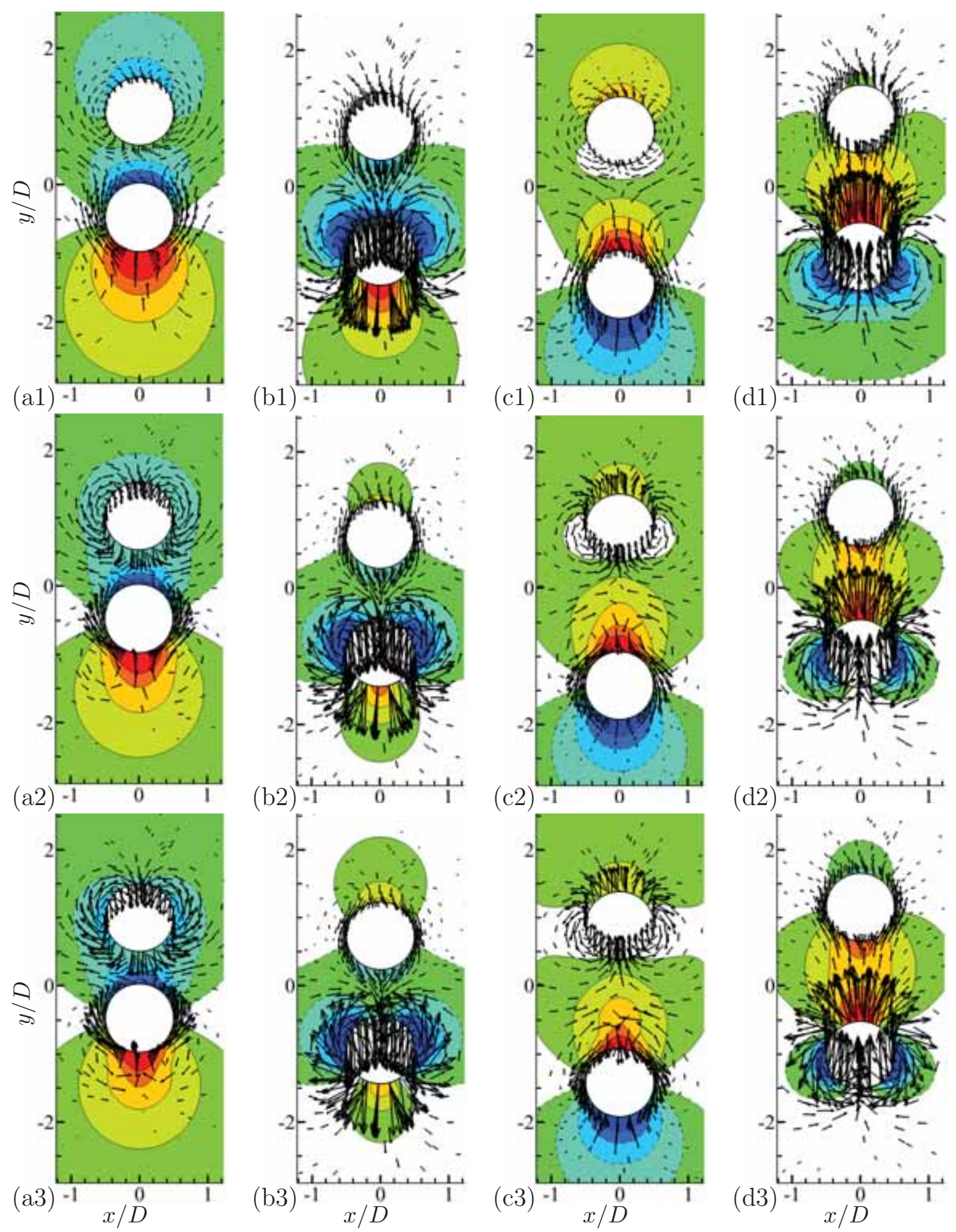

FIG. 18: Pressure coefficient contours and velocity vectors at $G / D=$ $0.9, A_{1} / D=0.477, m^{*}=2, \zeta=0$ and (1) $R e_{m}=10, f_{1} / f_{n}=0.7$, (2) $R e_{m}=50, f_{1} / f_{n}=0.745$, (3) $R e_{m}=110, f_{1} / f_{n}=0.765$, (a) $\phi_{1}=0^{\circ}$ (b) $\phi_{1}=90^{\circ}$ (c) $\phi_{1}=180^{\circ}$ (d) $\phi_{1}=270^{\circ}$. Primary resonance occurs at around $f_{1} / f_{n}=0.75$ and the damping factor is zero. 

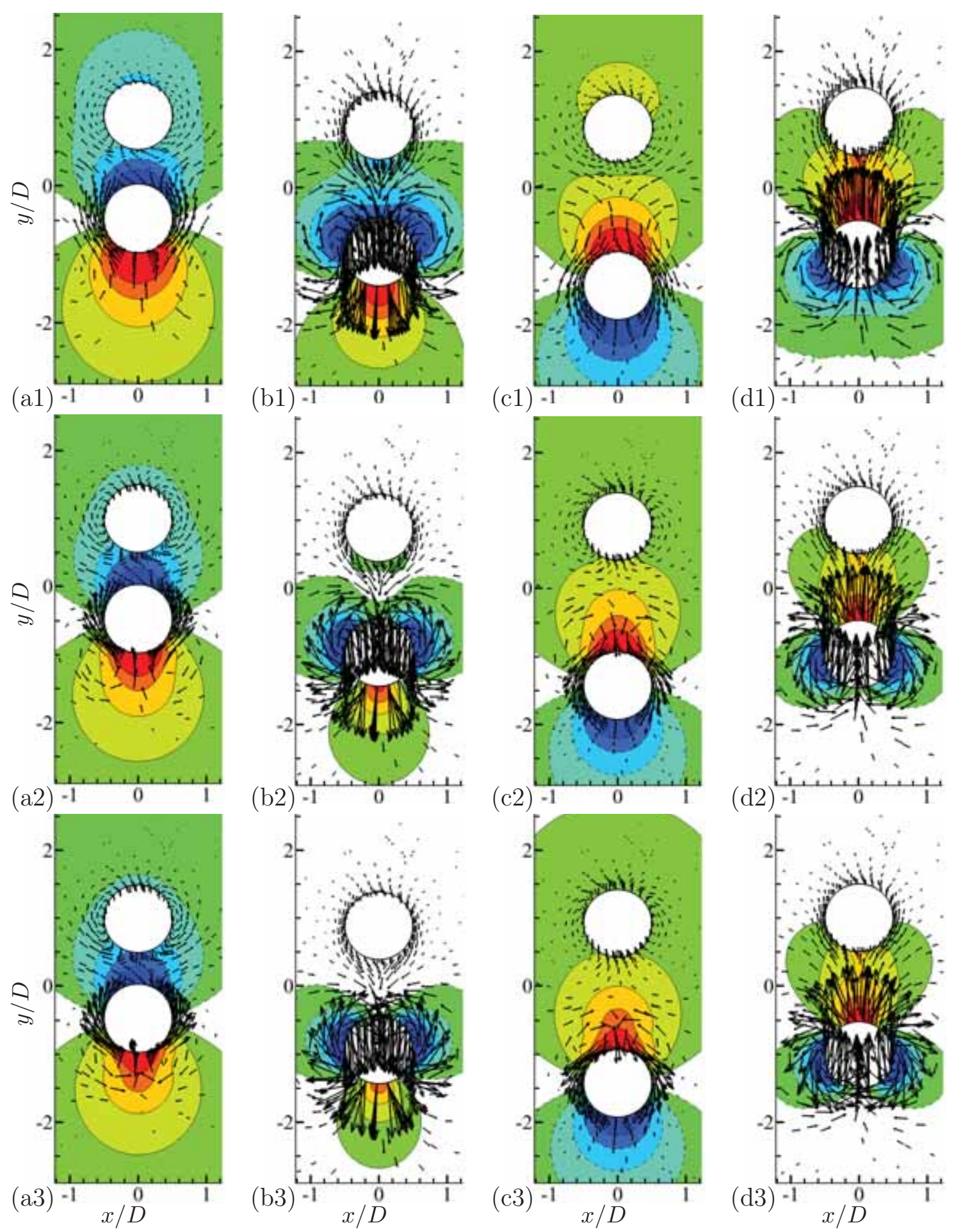

FIG. 19: Pressure coefficient contours and velocity vectors at $G / D=$ $0.9, A_{1} / D=0.477, m^{*}=2, \zeta=0.2$ and (1) $R e_{m}=10, f_{1} / f_{n}=0.73$, (2) $R e_{m}=50, f_{1} / f_{n}=0.775$, (3) $R e_{m}=110, f_{1} / f_{n}=0.795$, (a) $\phi_{1}=0^{\circ}$ (b) $\phi_{1}=90^{\circ}$ (c) $\phi_{1}=180^{\circ}$ (d) $\phi_{1}=270^{\circ}$. Primary resonance occurs at around $f_{1} / f_{n}=0.75$ and the damping factor is relatively high at $\zeta=0.2$. 

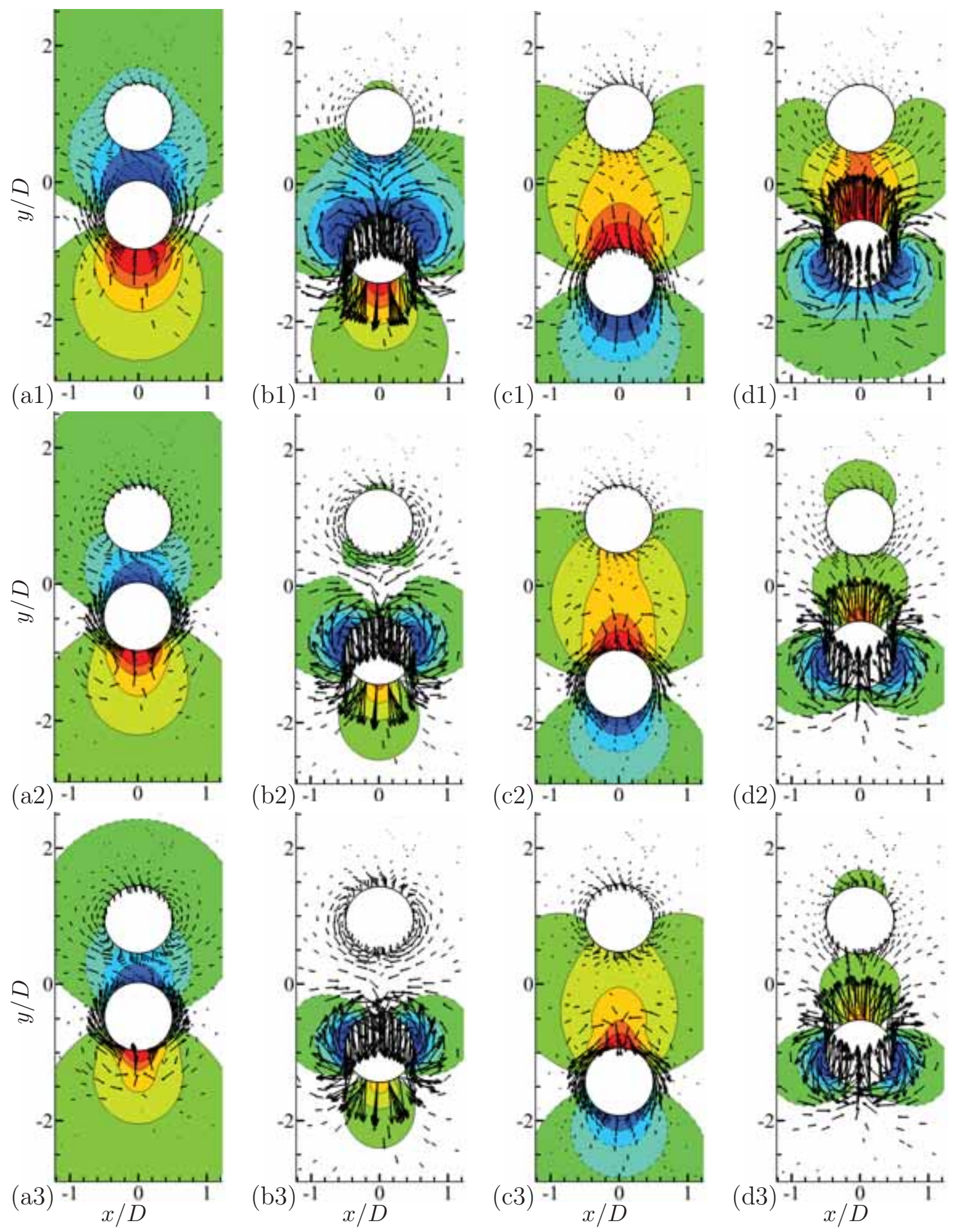

FIG. 20: Pressure coefficient contours and velocity vectors at $G / D=$ $0.9, A_{1} / D=0.477, m^{*}=2, \zeta=0, f_{1} / f_{n}=0.39$ and (1) $R e_{m}=10$, (2) $R e_{m}=50$, (3) $R e_{m}=110$, (a) $\phi_{1}=0^{\circ}$ (b) $\phi_{1}=90^{\circ}$ (c) $\phi_{1}=180^{\circ}$ (d) $\phi_{1}=270^{\circ}$. Secondary resonance occurs at $f_{1} / f_{n}=0.39$ and the damping factor is zero. 

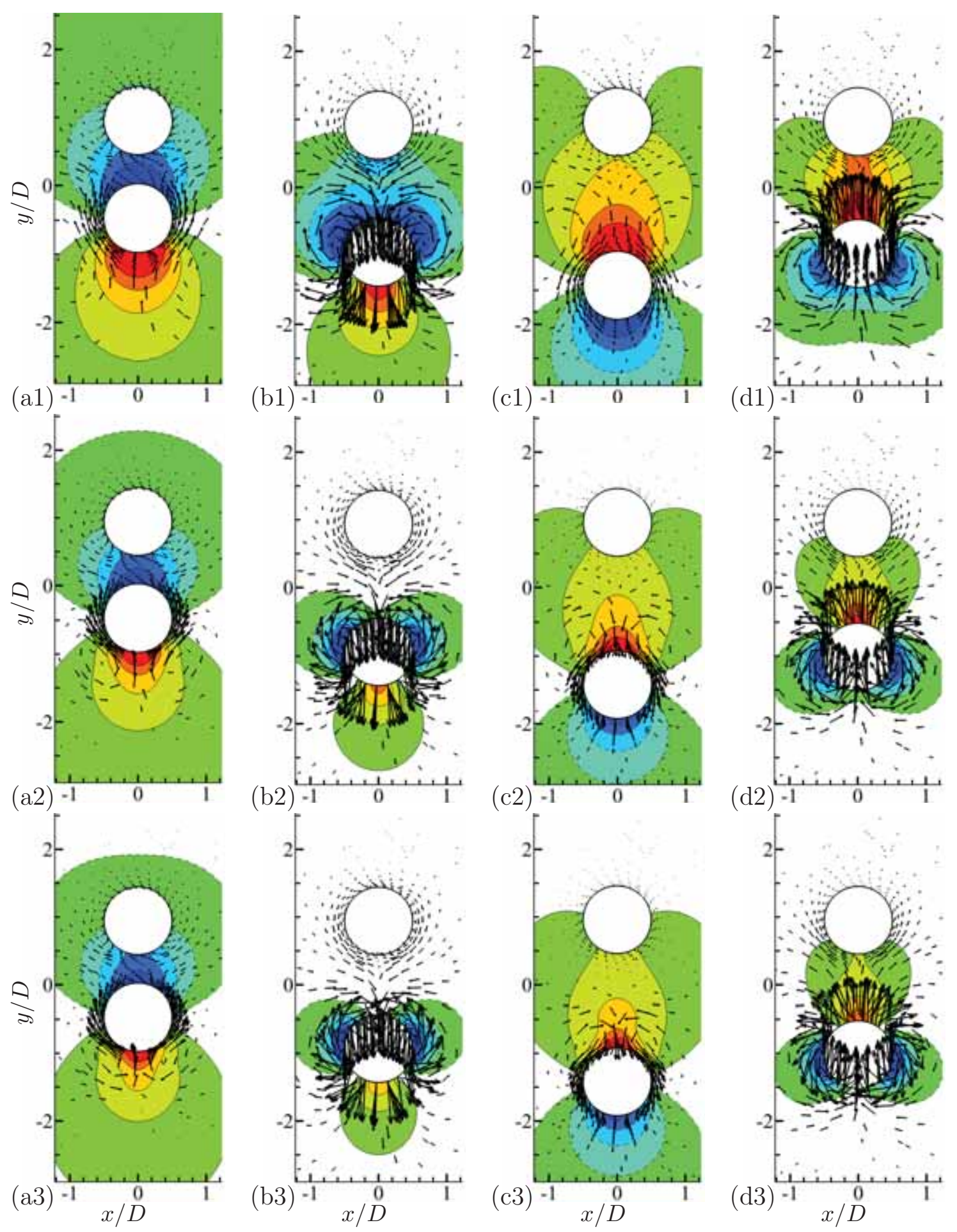

FIG. 21: Pressure coefficient contours and velocity vectors at $G / D=$ $0.9, A_{1} / D=0.477, m^{*}=2, \zeta=0.2, f_{1} / f_{n}=0.39$ and (1) $R e_{m}=10,(2)$ $R e_{m}=50$, (3) $R e_{m}=110$, (a) $\phi_{1}=0^{\circ}$ (b) $\phi_{1}=90^{\circ}$ (c) $\phi_{1}=180^{\circ}$ (d) $\phi_{1}=270^{\circ}$. Secondary resonance occurs at $f_{1} / f_{n}=0.39$ and the damping factor is relatively high at $\zeta=0.2$. 